\title{
Geometric Integrability of the Camassa-Holm Equation. II
}

\author{
Rafael Hernández Heredero ${ }^{1}$ and Enrique G. Reyes ${ }^{2}$ \\ ${ }^{1}$ Departamento de Matemática Aplicada, EUIT de Telecomunicación, \\ Universidad Politécnica de Madrid, Campus Sur, Ctra. de Valencia Km. \\ 7. 28031 Madrid, Spain and ${ }^{2}$ Departamento de Matemática y Ciencia de \\ la Computación, Universidad de Santiago de Chile, Casilla 307 Correo 2, \\ Santiago, Chile
}

Correspondence to be sent to: e-mail: ereyes@fermat.usach.cl

It is known that the Camassa-Holm (CH) equation describes pseudo-spherical surfaces and that therefore its integrability properties can be studied by geometrical means. In particular, the $\mathrm{CH}$ equation admits nonlocal symmetries of "pseudo-potential type": the standard quadratic pseudo-potential associated with the geodesics of the pseudospherical surfaces determined by (generic) solutions to $\mathrm{CH}$, allows us to construct a covering $\pi$ of the equation manifold of $\mathrm{CH}$ on which nonlocal symmetries can be explicitly calculated. In this article, we present the Lie algebra of (first-order) nonlocal $\pi$ symmetries for the $\mathrm{CH}$ equation, and we show that this algebra contains a semidirect sum of the loop algebra over $\operatorname{sl}(2, \mathbf{R})$ and the centerless Virasoro algebra. As applications, we compute explicit solutions, we construct a Darboux transformation for the $\mathrm{CH}$ equation, and we recover its recursion operator. We also extend our results to the associated Camassa-Holm equation introduced by J. Schiff. 


\section{Introduction}

The aim of this work is to continue our research on the geometric properties of the Camassa-Holm equation $[10,11]$

$$
2 u_{x} u_{x x}+u u_{x x x}=u_{t}-u_{x x t}+3 u_{x} u
$$

started in [43] (see also [44]). It was shown in that paper that the geometric approach introduced by Chern and Tenenblat in 1986 (see [12]) can be profitably applied to the study of Equation (1): The $\mathrm{CH}$ equation describes pseudo-spherical surfaces, and the analysis of their geodesics allows us to define a "Miura transform" and a "modified $\mathrm{CH}$ equation", which has been recently discussed in [22]. As a straightforward consequence. we can obtain infinite sequences of (local and nonlocal) conservation laws for (1). We remark that this construction was the first one which successfully generated an infinite number of nontrivial local conservation laws for the $\mathrm{CH}$ equation. Other constructions are in the original papers by Camassa and Holm [10, 11] (in which they generate explicitly three local conservation laws of $\mathrm{CH}$ using the bi-hamiltonian formalism), in [19] (in which conservation laws for $\mathrm{CH}$ are generated via hodographic transformations from conservation laws for the associated Camassa-Holm (ACH) equation introduced by Schiff in [49]), and in the very interesting work [33] (in which the bi-hamiltonian approach is effectively shown to generate an infinite number of local conservation laws).

The fact that the $\mathrm{CH}$ equation describes pseudo-spherical surfaces also yields, from purely geometric considerations, an $s l(2, \mathbf{R})$-valued linear problem associated with it, with reference to neither the bi-hamiltonian formulation of the equation as in [10], nor to its condition of being "dual" to the Korteweg-de Vries (KdV) equation as in Schiff's work [48]. The usual scalar linear problem associated with the $\mathrm{CH}$ equation [10,11] follows from the one considered in [43], as it does the one found by Schiff [48].

Finally, it was observed in [43] that the $\mathrm{CH}$ equation possesses a nonlocal symmetry depending essentially on a quadratic pseudo-potential naturally associated with the geodesics of the pseudo-spherical surfaces determined by $\mathrm{CH}$. It is well known that nonlocal symmetries are of interest: they carry information about the existence of linearizing transformations (Bluman and Kumei [9], Krasil'shchik and Vinogradov $[30,31,53]$ ) and Bäcklund/Darboux transformations (Krasil'shchik and Vinogradov [30, 31], Schiff [50], and Reyes [45]), and they also allow us to construct highly nontrivial 
families of solutions to the equations at hand (Galas [21], Schiff [50], Leo et al. [34, 35], and Reyes [45]). In this article, we continue the investigation of nonlocal symmetries of the Camassa-Holm equation: we construct an infinite-dimensional Lie algebra of nonlocal symmetries, we observe that it contains a semidirect sum of the loop algebra over $\operatorname{sl}(2, \mathbf{R})$ and the centerless Virasoro algebra, and as applications we obtain explicit solutions anda Darboux transformation, and we re-derive the $\mathrm{CH}$ recursion operator appearing in [20]. We note that in the case of the KdV equation, Guthrie [23] showed, using recursion operators, that KdV admits a Lie algebra of nonlocal symmetries isomorphic to a semidirect sum of the loop algebra over $\operatorname{sl}(2, \mathbf{R})$ and the Virasoro algebra with zero central charge. The semidirect sum appearing here is different from Guthrie's.

We also consider Schiff's ACH equation, we construct a Lie algebra of nonlocal symmetries (which, by reasons to be explained in the main text, is not exactly equal to the Lie algebra we find for $\mathrm{CH}$ ), and we present two Darboux transformations for it. We do not derive a recursion operator for $\mathrm{ACH}$ because its construction amounts to a simple repetition of our calculations for $\mathrm{CH}$.

We believe that the derivation of a Darboux transformation for $\mathrm{CH}$ appearing here is the first explicit construction of such a transformation for the CamassaHolm equation. On the other hand, a Darboux transform for ACH was considered before by Schiff in his paper [49]. The novelty of our approach rests on the fact that, while Schiff's transformation was originally obtained with the help of loop groups, we find it simply by using discrete symmetries of an equation associated with a quadratic pseudo-potential of $\mathrm{ACH}$, as in [12]. With respect to the $\mathrm{CH}$ recursion operator, it is well known that it can be constructed starting from the $\mathrm{CH}$ bihamiltonian structure $[10,11]$, or from the recursion operator for the KdV equation [20]. Instead, we find it exploiting the fact that one of our nonlocal symmetries is related to the "squared eigenfunctions" of the scalar linear problem associated to the $\mathrm{CH}$ equation.

We organize our work as follows: In Section 2, we review the theory of nonlocal symmetries of partial differential equations. We essentially follow Krasil'shchik and Vinogradov's approach [30,31], although we formulate their theory as explicitly as we can. We partially base this section on the expositions appearing in the papers $[45,46]$ by the second-named author. We then present Lie algebras of nonlocal symmetries for the $\mathrm{CH}$ and $\mathrm{ACH}$ equations in Section 3. Finally, in Section 4 we integrate these symmetries, we present examples of (smooth and nonsmooth) explicit solutions, and we obtain our recursion operator and Darboux transformations. Some of the results appearing here have been announced in the Fast Track Communication [26]. 
The following conventions will apply hereafter: Independent variables will be denoted by $x^{i}, i=1,2, \ldots, n$, and dependent variables by $u^{\alpha}, \alpha=1,2, \ldots, m ; k$-tuples $J=\left(j_{1}, \ldots, j_{k}\right), 0 \leq j_{1}, j_{2}, \ldots, j_{k} \leq n$ will denote multi-indices of order $\# J=k$; partial derivatives with respect to $x^{i}$ will be indicated by subindices; $D_{i}$ will stand for the total derivative with respect to $x^{i}$ :

$$
D_{i}=\frac{\partial}{\partial x^{i}}+\sum_{\alpha=1}^{m} \sum_{\# J_{00}} u_{J i}^{\alpha} \frac{\partial}{\partial u_{J}^{\alpha}}
$$

in which $u_{J i}^{k}=\partial u_{J}^{k} / \partial x^{i}$. Sometimes we will also use the Einstein summation convention. Familiarity with the geometric theory of classical and generalized symmetries as it appears in $[31,40]$ will be assumed throughout, but we recall here the following facts, which we shall use below:

(1) A (system of) differential equation(s) of order $l, \Xi_{a}=0, a=1, \ldots, k$, determines a subbundle $S^{(l)}$ of the $l$ th jet bundle $J^{l} E$, in which $E$ is the space of independent and dependent variables, $E=\mathbf{R}^{n} \times \mathbf{R}^{m}$.

(2) The bundle $J^{l} E$ and its subbundle $S^{(l)}$ can be prolonged an infinite number of times-assuming, as we always do, that the equation being considered satisfies a technical condition dubbed "formal integrability" by Goldschmidt, see [31] and references therein-giving rise to the bundle of infinite jets $J^{\times} E$ and to the subbundle $S^{(\infty)}$ of $J^{\infty} E$. This subbundle is known as the equation manifold of $\Xi_{a}=0$.

(3) The total derivatives (2) generate the Cartan distribution $\mathcal{C}$ on $J^{\infty} E$. Dually, we can define $\mathcal{C}$ using differential forms: we say that a differential form $\omega$ on $J^{\aleph} E$ is a contact form if $j^{\aleph}(s)^{*} \omega=0$ for any local section $s$ of $E$, in which $j \sim(s)$ is the infinite prolongation of $s$. The set of contact forms is a differential ideal of the ring of differential forms on $J^{\infty} E$, locally generated by the basic contact 1 -forms

$$
\theta_{i_{1} \ldots i_{k}}^{\alpha}=\mathrm{d} u_{i_{1} \ldots i_{k}}^{\alpha}-\sum_{j} u_{i_{1} \ldots i_{k} j}^{\alpha} \mathrm{d} x^{j}, \quad k \geq 0 .
$$

The distribution $\mathcal{C}$ can be characterized as the space of all vector fields on $J^{\infty} E$ annihilated by the space of contact l-forms. Since $\mathrm{d} \theta_{i_{1} \ldots i_{k}}^{\alpha}=\sum_{j} \mathrm{~d} x^{j} \wedge$ $\theta_{i_{1} \ldots i_{k}, j}^{\alpha}$, the distribution $\mathcal{C}$ is involutive.

(4) The Cartan distribution on $J^{\infty} E$ pull-backs to the equation manifold $S^{(\infty)}$, giving rise to an involutive distribution $\mathcal{C}\left(S^{(\infty)}\right)$ on $S^{(\infty)}$. Following standard 
practice, we continue denoting the pull-backs of the total derivatives (i.e., the local generators of the distribution $\left.\mathcal{C}\left(S^{(\varkappa)}\right)\right)$ as $D_{i}$.

\section{Nonlocal Symmetries of Partial Differential Equations}

Nonlocal symmetries of (systems of) partial differential equations were first studied rigorously from a geometric point of view by Vinogradov and Krasil'shchik in their seminal paper [53], and full accounts of their research appear in [30, 31]. Other approaches to nonlocal symmetries (of formal, algebraic, or heuristic nature) have also been advanced in the literature (see, e.g.. [1, 2, 9, 41, 42]), but we believe that the Krasil'shchikVinogradov geometric viewpoint remains the most satisfactory approach to nonlocalities at our disposal. We mention three interesting earlier references on applications of the Krasil'shchik-Vinogradov theory: van Bemmelen [6], Dodd [18], and Kirnasov [29].

Definition 1. Let $N$ be a nonzero integer or $N=\infty$. An $N$-dimensional covering $\pi$ of a (system of) partial differential equation(s) $\Xi_{a}=0, a=1, \ldots, k$, is a triplet

$$
\left(\left\{\gamma^{b}: b=1, \ldots, N\right\} ;\left\{X_{i b}: b=1, \ldots, N ; i=1, \ldots, n\right\} ;\left\{\bar{D}_{i}: i=1, \ldots, n\right\}\right)
$$

of variables $\gamma^{b}$, smooth functions $X_{i b}$ depending on $x^{i}, u^{\alpha}, \gamma_{b}$ and a finite number of partial derivatives of $\boldsymbol{u}^{k}$, and linear operators

$$
\tilde{D}_{i}=D_{i}+\sum_{b=1}^{N} X_{i b} \frac{\partial}{\partial \gamma^{b}},
$$

such that the equations

$$
\tilde{D}_{i}\left(X_{j b}\right)=\bar{D}_{j}\left(X_{i b}\right), \quad i, j=1,2, \ldots, n, b=1,2, \ldots, N
$$

hold whenever $u^{\alpha}\left(x^{i}\right)$ is a solution to $\Xi_{a}=0$.

Smooth functions such as $X_{i b}$, depending on variables $x^{i}, u^{\alpha}$, a finite number of derivatives of $u^{\alpha}$, and the new variables $\gamma^{b}$, will be called differential functions, generalizing slightly the conventions of [40]. It will be understood hereafter that the indices $i$ and $b$ run from 1 to $n$ and from 1 to $N$, respectively, so that instead of (4) we simply write $\pi=\left(\gamma^{b} ; X_{i b} ; \tilde{D}_{i}\right)$. The variables $\gamma^{b}$ are interpreted as new dependent variables-the "nonlocal variables" of the theory-the number $N$ is the dimension of the covering, and 
the operators $\tilde{D}_{i}$ satisfying Equation (6) are new total derivatives naturally extending the operators $D_{i}$ on $S^{(\infty)}$.

The covering $\pi$ can be thought of as a fiber bundle over $S^{(\varkappa)}$, the vertical coordinates being precisely the variables $\gamma^{b}$. We formalize this view stating that a covering $\pi$ consists of a manifold of the form $S^{(\infty)} \times \mathbf{R}^{N}$ equipped with a distribution $\tilde{C}$ (the distribution generated by the vector fields $\tilde{D}_{i}$ ), which projects on to the Cartan distribution of $S^{(\infty)}$. Equation (6) implies that $\left[\tilde{D}_{i}, \tilde{D}_{j}\right]=0$, and therefore $\widetilde{C}$ is involutive. The general theory of manifolds such as $S^{(\infty)} \times \mathbf{R}^{N}$ can be extracted from [7, 31], and several examples of coverings appear in $[6,29]$.

Now the operators $\tilde{D}_{i}$ satisfy $\tilde{D}_{i}\left(\gamma^{b}\right)=X_{i b}$, and these equations are compatible because (6) holds. Since we expect that on solutions to the system of equations $\Xi_{a}=0$ the total derivatives $\bar{D}_{i}$ become standard partial derivatives, the equations

$$
\frac{\partial \gamma^{b}}{\partial X^{i}}=X_{i b}
$$

should hold for each index $b$ whenever $u^{\alpha}\left(x^{i}\right)$ is a solution to $\Xi_{a}=0$. These compatible equations specify the relations between the dependent variables $u^{\alpha}$ and the new dependent variables $\gamma^{b}$. Conversely, a set of equations of the form (7), which is compatible on solutions to the system $\Xi_{a}=0$, determines a covering $\pi=\left(\gamma^{b} ; X_{i b} ; \tilde{D}_{i}\right)$ where the differential operators $\tilde{D}_{i}$ are defined by means of (5).

Equations (7) are crucial, as they allow us to study nonlocal symmetries basically via the local theory appearing, for example, in [40]:

Let us assume that the dimension $N$ of the covering is finite (not a serious restriction for us, since the case $N=\infty$ will not appear in the explicit computations to be presented in subsequent sections). We consider the bundle $\bar{E}=E \times \mathbf{R}^{N}$ equipped with local coordinates $\left(x^{i}, u^{\alpha}, \gamma^{b}\right)$, and form the infinite jet space $J^{\varkappa} \bar{E}$ equipped with canonical coordinates

$$
\left(x^{i}, u^{\alpha}, u_{j_{1}}^{\alpha}, u_{j_{1}, j_{2}}^{\alpha}, \ldots, u_{J}^{\alpha}, \ldots, \gamma^{b}, \gamma_{i_{1}}^{b}, \gamma_{i_{1}, i_{2}}^{b}, \ldots, \gamma_{I}^{b}, \ldots\right)
$$

in which $I$ and $J$ are multi-indices and $\gamma_{i}^{c}=\partial \gamma^{c} / \partial x^{i}$, etc. If

$$
X=\xi^{i} \frac{\partial}{\partial x^{i}}+\phi^{\alpha} \frac{\partial}{\partial u^{\alpha}}+\varphi^{b} \frac{\partial}{\partial y^{b}}
$$

is a (generalized) vector field on $\bar{E}$, we can prolong it to a vector field on $J^{\varkappa} \bar{E}$ by means of the standard prolongation formula [40] as follows. Let us denote by $\bar{D}_{i}$ the total 
derivative operators on $J^{\infty} \bar{E}$; then the infinite prolongation $\operatorname{pr}^{\nsim} X$ of $X$ is given by

$$
\operatorname{pr}^{\infty} X=X+\phi_{J}^{\alpha} \frac{\partial}{\partial u_{J}^{\alpha}}+\varphi_{I}^{b} \frac{\partial}{\partial \gamma_{I}^{b}}
$$

in which we sum over all multi-indices $I, J$ of order greater than or equal to 1 , and the coefficients $\phi_{J}^{\alpha}$ and $\varphi_{I}^{b}$ are given inductively by

$$
\begin{aligned}
& \phi_{0}^{\alpha}=\phi^{\alpha} ; \quad \phi_{J i}^{\alpha}=\bar{D}_{i} \phi_{J}^{\alpha}-\sum_{k=1}^{n} \bar{D}_{i}\left(\xi^{k}\right) u_{J k}^{\alpha} ; \\
& \varphi_{0}^{b}=\varphi^{b} ; \quad \varphi_{I i}^{b}=\bar{D}_{i} \varphi_{I}^{b}-\sum_{k=1}^{n} \bar{D}_{i}\left(\xi^{k}\right) \gamma_{I k}^{b} .
\end{aligned}
$$

The following is our definition of nonlocal symmetries (Cf. [31, pp. 249-250]).

Definition 2. Let $\Xi_{a}=0, a=1 \ldots, k$, be a system of partial differential equations, $\pi=$ $\left(\gamma^{b} ; X_{i b}: \bar{D}_{i}\right)$ be a covering of $\Xi_{a}=0$, and $\bar{E}=E \times \mathbf{R}^{N}$ be the bundle defined above. A nonlocal $\pi$-symmetry of $E_{a}=0$ is a generalized vector field

$$
X=\xi^{i} \frac{\partial}{\partial X^{i}}+\phi^{\alpha} \frac{\partial}{\partial u^{\alpha}}+\varphi^{b} \frac{\partial}{\partial y^{b}}
$$

on $\bar{E}$ such that

$$
\operatorname{pr}^{\infty} X\left(\Xi_{a}\right)=0, \quad \text { and } \operatorname{pr}^{\infty} X\left(\frac{\partial y^{b}}{\partial X^{i}}-X_{i b}\right)=0
$$

on solutions to the augmented system

$$
\Xi_{a}=0 ; \quad \frac{\partial \gamma^{b}}{\partial x^{i}}=X_{i b}
$$

Clearly, this definition generalizes exactly the standard local case as considered, for instance, in [40]. Now, as explained in [40], it is enough to consider evolutionary generalized vector fields in order to capture all possible generalized symmetries of the augmented system (11). Thus, hereafter we shall consider only generalized vector fields $X$ of the form

$$
X=\sum_{\alpha} G^{\alpha} \frac{\partial}{\partial u^{\alpha}}+\sum_{b} H^{b} \frac{\partial}{\partial y^{b}},
$$

in which $G^{\alpha}$ and $H^{b}$ are smooth functions on $J^{\infty} \bar{E}$. The symmetry condition (10) can be further simplified because we are allowed to replace all derivatives of $\gamma^{b}$ appearing in 
$G^{\alpha}$ and $H^{b}$ by means of the equations

$$
\frac{\partial \gamma^{b}}{\partial X^{i}}=X_{i b}
$$

and their derivatives. Explicitly, if $X$ is given by (12), the symmetry conditions (10), on solutions to the augmented system (11), become

$$
0=\operatorname{pr}^{\cdots} X\left(\Xi_{a}\right)=\sum_{\alpha, J}\left(\bar{D}_{J} G^{\alpha}\right) \frac{\partial \Xi_{a}}{\partial u_{J}^{\alpha}}=\sum_{\alpha, J} \frac{\partial \Xi_{a}}{\partial u_{J}^{\alpha}} \bar{D}_{J}\left(G^{\alpha}\right)
$$

and

$$
\begin{aligned}
0=\operatorname{pr}^{\infty} X\left(\gamma_{i}^{c}-X_{i c}\right) & =\sum_{\alpha, J}\left(\bar{D}_{J} G^{\alpha}\right) \frac{\partial}{\partial u_{J}^{\alpha}}\left(\gamma_{i}^{c}-X_{i c}\right)+\sum_{b, I}\left(\bar{D}_{I} H^{b}\right) \frac{\partial}{\partial \gamma_{I}^{b}}\left(\gamma_{i}^{c}-X_{i c}\right) \\
& =-\sum_{\alpha, J}\left(\bar{D}_{J} G^{\alpha}\right) \frac{\partial X_{i c}}{\partial u_{J}^{\alpha}}+\tilde{D}_{i} H^{c}-\sum_{b} H^{b} \frac{\partial X_{i c}}{\partial \gamma^{b}} .
\end{aligned}
$$

If we define the matrix $\tilde{\xi}_{*}$ as the standard formal linearization of the system $\Xi_{a}=0$ "lifted" to the covering $\pi$ (see $[30,31]$ and references therein), that is.

$$
\dot{\Xi}_{*}=\left(\sum_{L} \frac{\partial \Xi_{a}}{\partial u_{L}^{\alpha}} \tilde{D}_{L}\right)=\left(\begin{array}{ccc}
\frac{\partial \Xi_{1}}{\partial u^{1}}+\cdots+\frac{\partial \Xi_{1}}{\partial u_{I}^{1}} \tilde{D}_{I} & \frac{\partial \Xi_{1}}{\partial u^{2}}+\cdots+\frac{\partial \Xi_{1}}{\partial u_{J}^{2}} \tilde{D}_{J} & \ldots \\
\frac{\partial \Xi_{2}}{\partial u^{1}}+\cdots+\frac{\partial \Xi_{2}}{\partial u_{K}^{l}} \tilde{D}_{K} & \ldots & \ldots \\
\vdots & &
\end{array}\right)
$$

and we also set

$$
\tilde{D}_{\tau}=\sum_{\alpha=1}^{m} \sum_{\# Z K^{\prime}} \tilde{D}_{K}\left(G^{\alpha}\right) \frac{\partial}{\partial u_{K}^{\alpha}}+\sum_{c=1}^{N} H^{c} \frac{\partial}{\partial \gamma^{c}},
$$

we can summarize the foregoing remarks as follows:

Proposition 1. Let $\Xi_{a}=0, a=1, \ldots, k$, be a system of partial differential equations and let $\pi=\left(\gamma^{b} ; X_{i b} ; \bar{D}_{i}\right)$ be a covering of $\Xi_{a}=0$. Assume, without loss of generality, that

$$
X=\sum_{\alpha} G^{\alpha} \frac{\partial}{\partial u^{\alpha}}+\sum_{b} H^{b} \frac{\partial}{\partial \gamma^{b}}
$$

in which $G^{\alpha}$ and $H^{b}$ are differential functions on $S^{(\infty)} \times \mathbf{R}^{N}$, and set

$$
G=\left(G^{1}, G^{2}, \ldots, G^{m}\right)^{t}
$$


Then, $X$ is a nonlocal $\pi$-symmetry of $\Xi_{a}=0$ if and only if the equations

$$
\begin{aligned}
\tilde{\Xi}_{*}(G) & =0, \quad \text { and } \\
\tilde{D}_{i}\left(H^{b}\right) & =\tilde{D}_{\tau}\left(X_{i b}\right),
\end{aligned}
$$

hold on solutions to the augmented system (11).

Thus, if the "infinitesimal deformation" of the variables $u^{\alpha}$ along $X$ is measured by $G$, and the corresponding deformation of the nonlocal variables $\gamma^{b}$ is measured by the functions $H^{b}$ (i.e., $u_{\mathrm{\tau}}^{\alpha}=G^{\alpha}$ and $\gamma_{\tau}^{b}=H^{b}$ ), then heuristically Equations (18) and (19) tell us that $X$ is a nonlocal $\pi$-symmetry of the system $\Xi_{a}=0$ if and only if the linearized equations

$$
\frac{\partial}{\partial \tau} \Xi_{a}=0 \text { and } \frac{\partial}{\partial \tau}\left(\frac{\partial \gamma^{b}}{\partial X^{i}}\right)=X_{i b, \tau}
$$

are satisfied whenever $u^{\alpha}\left(x^{i}\right)$ and $y^{b}\left(x^{i}\right)$ are solutions to the system (11).

Note that Equation (18) depends only on the vector $G$ and the system $\Xi_{a}=0$, and that it is the obvious "nonlocal generalization" of the condition for local symmetries $[1,2,9,31,40]$. Following Krasil'shchik and Vinogradov [30, 31, 53], we call $G$ the $\pi$-shadow of the nonlocal $\pi$-symmetry $X$. We also remark that nonlocal $\pi$-symmetries form a Lie algebra, and that this structure exists because we work on a fixed covering of the equation at hand. We do not expect the "space of all nonlocal symmetries" of a given equation to possess a Lie algebra structure. This important point is discussed in [30] and also in [41,42], in which a proposal for transforming a "space of nonlocal evolutionary vector fields" into a Lie algebra is made.

Hereafter, we assume that a covering (4) of $\Xi_{a}=0$ has been fixed, and we simply say "nonlocal symmetry" instead of "nonlocal $\pi$-symmetry". Since generalized symmetries transform solutions into solutions $[31,40]$, we have the following important result.

Corollary 1. If $u_{0}^{\alpha}\left(x^{i}\right)$ and $\gamma_{0}^{b}\left(x^{i}\right)$ are solutions to the augmented system (11), the solution to the Cauchy problem

$$
\begin{aligned}
\frac{\partial u^{\alpha}}{\partial \tau} & =G^{\alpha}, \quad \frac{\partial \gamma^{b}}{\partial \tau}=H^{b} ; \\
u^{\alpha}\left(x^{i}, 0\right) & =u_{0}^{\alpha}\left(x^{i}\right), \quad \gamma^{b}\left(x^{i}, 0\right)=\gamma_{0}^{b}\left(x^{i}\right)
\end{aligned}
$$


is a one-parameter family of solutions to the augmented system (11). In particular, nonlocal symmetries send solutions to the system $\Xi_{a}=0$ to solutions of the same system.

\section{Nonlocal Symmetries for the CH and ACH Equations}

As pointed out by Krasil'shchik, Vinogradov, and their coworkers (see [30, 31] and references therein), standard Bäcklund transformations, zero curvature representations, conservation laws, Wahlquist-Eastbrook pseudo-potentials, and pseudo-differential recursion operators give rise to coverings and so, we may expect, to nonlocal symmetries; see, for instance, [2, 6, 9, 21, 23, 24, 29-31]. The symmetries presented below depend on pseudo-potentials of the equations at hand, which we introduce as follows:

Definition 3. Let $\Xi=0$ be a scalar partial differential equation in two independent variables. A function $\Gamma$ is a pseudo-potential for $\Xi=0$ if there exist smooth functions $f$ and $g$ depending on $x, t, u$, a finite number of derivatives of $u$ and $\Gamma$, such that the 1 -form

$$
\Omega_{\Gamma}=\mathrm{d} \Gamma-(f \mathrm{~d} x+g \mathrm{~d} t),
$$

satisfies

$$
\mathrm{d} \Omega_{\Gamma}=0 \bmod \Omega_{\Gamma}
$$

whenever $u(x, t)$ is a solution to $\Xi=0$.

In other words, $\Gamma$ is a pseudo-potential for $\Xi=0$ if and only if $f_{t}=g_{X}$ whenever $\Gamma_{x}=f, \Gamma_{t}=g$ and $u(x, t)$ is a solution to $\Xi=0$. Of course, if the functions $f$ and $g$ above do not depend on $\Gamma$ at all, $\Omega_{\Gamma}$ is a local conservation law for $\Xi=0$ with potential $\Gamma$. Definition 3 is motivated by the seminal work of Wahlquist and Eastbrook [54] on quadratic pseudo-potentials of the KdV equation. Analogous definitions have been given by Sasaki [47] and Molino [38].

\subsection{Nonlocal symmetries of the $\mathrm{CH}$ equation}

We now consider nonlocal symmetries of the Camassa-Holm equation

$$
2 u_{x} u_{x x}+u u_{x x x}=u_{t}-u_{x x t}+3 u_{x} u
$$

which we write as a system of equations for two dependent variables $m$ and $u$

$$
m=u_{\mathrm{xx}}-u, \quad m_{t}=-m_{x} u-2 m u_{\mathrm{x}} .
$$


Our first remark [43] is that we can find a pseudo-potential $\gamma$ for the $\mathrm{CH}$ equation and also two potentials for conservation laws.

Theorem 1. The system of equations

$$
\gamma_{x}=m-\frac{1}{2 \lambda} \gamma^{2}+\frac{1}{2} \lambda, \quad \gamma_{t}=\lambda\left(u_{x}-\gamma-\frac{1}{\lambda} u \gamma\right)_{x}
$$

in which $\lambda$ is a nonzero real parameter, is completely integrable on solutions to $\mathrm{CH}$, and therefore it determines a pseudo-potential $\gamma$ for the $\mathrm{CH}$ equation. Moreover, the following two systems of equations are compatible on solutions to (23) and therefore they define potentials $\delta$ and $\beta$ for (nonlocal) conservation laws of the $\mathrm{CH}$ equation:

$$
\delta_{x}=\gamma, \quad \delta_{t}=\lambda\left(u_{x}-\gamma-\frac{1}{\lambda} u \gamma\right)
$$

and

$$
\beta_{X}=m \mathrm{e}^{s / \lambda}, \quad \beta_{t}=\mathrm{e}^{\delta / \lambda}\left[-(1 / 2) \gamma^{2}+(1 / 2) \lambda^{2}-u m\right]
$$

The pseudo-potential $\gamma$ can be understood geometrically in terms of geodesics of the pseudo-spherical surfaces associated with the $\mathrm{CH}$ equation (23); see [12] and the later work [22]. A geometric derivation of (24) appears in [43], but of course Theorem 1 can be checked directly. Its importance for us rests on the fact that the compatible system of Equations (24)-(26) yields a three-dimensional covering $\pi$ of the Camassa-Holm equation.

Remark 1. As explained in [43] (see also [22, 44]), Theorem 1 allows us to generate conservation laws for the $\mathrm{CH}$ equation. Indeed, the second equation appearing in (24) encodes an infinite number of local conservation laws for $\mathrm{CH}$ : if we write the function $\gamma$ as $\gamma=\sum_{n=1}^{\infty} \gamma_{n} \lambda^{n / 2}$, we can find the functions $\gamma_{n}$ in terms of $u, m$, and their $x$-derivatives recursively, using the first equation appearing in (24). If we then replace back into the second equation of (24), we obtain a sequence of local conservation laws for $\mathrm{CH}$, as claimed. Their nontriviality is analyzed in [43]. We can also set $\gamma=\lambda+\sum_{n=0}^{\infty} \gamma_{n} \lambda^{-n}$. This expansion yields three local conservation laws and a sequence of nonlocal conservation laws (in the sense that their conserved densities and fluxes are defined on coverings of the $\mathrm{CH}$ equation). We once again refer to $[22,43]$ for details. These comments imply that the well-known fact that smooth solutions to the Camassa-Holm equation can develop 
singularities in finite time $[13,15,36]$ is not due to the lack of local conserved quantities, as suspected for instance in [36]. Rather, the point is that the local conserved quantities do not guarantee the existence of a priori bounds for the first derivative of the solutions $u(x, t)$ to the $\mathrm{CH}$ equation. A careful analysis of the behavior of $u_{x}$ in peakon-antipeakon collisions appears in [5]. Finally, we note that Equations (26) determine a nonlocal conservation law on a covering equipped with nonlocal variables $\gamma$ and $\delta$. The existence of this conservation law is essentially connected with nonlocal symmetries; see Remark 2 below.

We now classify all first-order nonlocal $\pi$-symmetries of the $\mathrm{CH}$ equation. We stress that we do not find "all" nonlocal symmetries of the $\mathrm{CH}$ equation (23). We believe we cannot expect to do so, due to the ease with which we can construct nonlocal objects. However, given some a priori restrictions, it is possible to obtain interesting classifications, as we show below. We also mention that classifications of nonlocal symmetries (for Burgers' equation and for a class of nonlinear heat equations) can be found in $[29,31]$.

Now, we certainly expect that our classification recovers, in particular, all known local classical symmetries of $\mathrm{CH}$. In order to do this (concretely, in order to recover the scaling symmetry $t \partial / \partial t-u \partial / \partial u$ of $\mathrm{CH}$ ), we find it necessary to assume that the parameter $\lambda$ appearing in (24)-(26) is also affected by the symmetry transformation. In other words, we extend the augmented system (23)-(26) with the equations

$$
\lambda_{x}=0, \quad \lambda_{t}=0,
$$

and we consider evolutionary vector fields of the form

$$
V=G^{1} \frac{\partial}{\partial m}+G^{2} \frac{\partial}{\partial u}+H^{1} \frac{\partial}{\partial y}+H^{2} \frac{\partial}{\partial \delta}+H^{3} \frac{\partial}{\partial \beta}+K \frac{\partial}{\partial \lambda}
$$

in which $G^{\alpha}, H^{b}$, and $K$ are functions of $x, t, m, u, \gamma, \delta, \beta, \lambda$, and first derivatives of $m$ and $u$.

Theorem 2. The first-order generalized symmetries of the augmented $\mathrm{CH}$ system (23)-(27), represented by vector fields (28), are

$$
\begin{aligned}
V_{1}= & \left(2 m u_{X}+u m_{X}\right) \frac{\partial}{\partial m}-u_{t} \frac{\partial}{\partial u}+\left(\frac{\lambda^{2}}{2}-\frac{\lambda u}{2}+u m-\frac{\gamma^{2}}{2}-\frac{u \gamma^{2}}{2 \lambda}+\gamma u_{X}\right) \frac{\partial}{\partial \gamma} \\
& +\left(\lambda \gamma+u \gamma-\lambda u_{X}\right) \frac{\partial}{\partial \delta}-\frac{1}{2} e^{s / \lambda}\left(\lambda^{2}-2 u m-\gamma^{2}\right) \frac{\partial}{\partial \beta},
\end{aligned}
$$




$$
\begin{aligned}
& V_{2}=m_{x} \frac{\partial}{\partial m}+u_{x} \frac{\partial}{\partial u}+\left(\frac{\lambda}{2}+m-\frac{\gamma^{2}}{2 \lambda}\right) \frac{\partial}{\partial \gamma}+\gamma \frac{\partial}{\partial \delta}+\mathrm{e}^{\delta / \lambda} m \frac{\partial}{\partial \beta}, \\
& V_{3}=\frac{\partial}{\partial \delta}+\frac{\beta}{\lambda} \frac{\partial}{\partial \beta}, \\
& V_{4}=\frac{\partial}{\partial \beta}, \\
& V_{5}=\mathrm{e}^{\delta / \lambda}\left(\frac{2 m \gamma}{\lambda}+m_{X}\right) \frac{\partial}{\partial m}+\mathrm{e}^{\delta / \lambda} \gamma \frac{\partial}{\partial u}+\mathrm{e}^{\delta / \lambda} m \frac{\partial}{\partial \gamma}+\beta \frac{\partial}{\partial \delta}+\left(\mathrm{e}^{2 \delta / \lambda} m+\frac{\beta^{2}}{2 \lambda}\right) \frac{\partial}{\partial \beta}, \\
& V_{6}=A(\lambda)\left(m \frac{\partial}{\partial m}+u \frac{\partial}{\partial u}+\gamma \frac{\partial}{\partial \gamma}+\delta \frac{\partial}{\partial \delta}+\beta \frac{\partial}{\partial \beta}+\lambda \frac{\partial}{\partial \lambda}-t V_{1}\right),
\end{aligned}
$$

where $A(\lambda)$ is an arbitrary function. Consequently, these vector fields are nonlocal $\pi$ symmetries of the $\mathrm{CH}$ equation.

This classification has been found via extensive symbolic computations using Mathematica software written by the authors [25]. Note that the function $A(\lambda)$ is included in $V_{6}$ since it affects the way in which $\lambda$ varies with the infinitesimal symmetry transformation (34). This function is also of importance for our observations on the Lie algebra structure of nonlocal $\pi$-symmetries; see Corollary 2 and Proposition 2 below. We also note that $V_{1}$ and $V_{2}$ are simply the generators of shifts with respect to the independent variables: they are equivalent to $\partial / \partial t$ and $-\partial / \partial x$, respectively.

Remark 2. Now we can explain the origin of the nonlocal conservation law

$$
\left(m \mathrm{e}^{\delta / \lambda}\right)_{t}=\left(\mathrm{e}^{\delta / \lambda}\left(-(1 / 2) \gamma^{2}+(1 / 2) \lambda^{2}-u m\right)\right)_{X},
$$

with potential $\beta$ given by Equations (26): We can see from (33) that the vector field $\left(\gamma \mathrm{e}^{\delta / \lambda}\right) \partial / \partial u$ is the shadow of a nonlocal symmetry, and (33) also tells us that the infinitesimal variation of the potential $\delta$ as $u$ changes to $u+\tau\left(\gamma \mathrm{e}^{\delta / \lambda}\right)$ is precisely $\beta$. Thus, this nonlocal conservation law is essentially a Lie derivative.

Corollary 2. The commutation table of the six nonlocal symmetries (29)-(34) of the $\mathrm{CH}$ equation is that of Figure 1 , whenever $u, m, \gamma, \delta, \beta, \lambda$ solve the augmented CH system (23)(27). 


\begin{tabular}{c||r|r|r|r|r|c|} 
& $V_{1}$ & $V_{2}$ & $V_{3}$ & $V_{4}$ & $V_{5}$ & $V_{6}{ }^{*}$ \\
\hline \hline$V_{1}$ & & & & & & $-B(\lambda) V_{1}$ \\
\hline$V_{2}$ & & & & & & \\
\hline$V_{3}$ & & & & $-\frac{1}{\lambda} V_{4}$ & $\frac{1}{\lambda} V_{5}$ & $B(\lambda) V_{3}$ \\
\hline$V_{4}$ & & & $\frac{1}{\lambda} V_{4}$ & & $V_{3}$ & $B(\lambda) V_{4}$ \\
\hline$V_{5}$ & & & $-\frac{1}{\lambda} V_{5}$ & $-V_{3}$ & & \\
\hline$V_{6}$ & $A(\lambda) V_{1}$ & & $-A(\lambda) V_{3}$ & $-A(\lambda) V_{4}$ & & $-\left[A^{\prime}(\lambda) B(\lambda)-A(\lambda) B^{\prime}(\lambda)\right] \frac{\lambda}{A(\lambda)} V_{6}$ \\
\hline
\end{tabular}

Fig. 1. The commutation table of the $\mathrm{CH}$ nonlocal symmetry algebra. In $V_{6}{ }^{*}$ we have used $B(\lambda)$ instead of $A(\lambda)$.

Remark 3. We stress the fact that the commutation table of Corollary 2 is valid only on shell. For example, the commutator of $V_{5}$ and $V_{6}$ is (we write $A, A^{\prime}, A^{\prime \prime}$, instead of $A(\lambda)$, $\left.A^{\prime}(\lambda), A^{\prime \prime}(\lambda)\right)$

$$
\begin{aligned}
{\left[V_{5}, V_{6}\right]=} & -e^{s / \lambda} A^{\prime} \lambda_{x}\left(m+t m_{t}\right) \frac{\partial}{\partial m} \\
& -e^{\delta / \lambda}\left(A^{\prime \prime} u \lambda_{x}^{2}+2 A^{\prime} \lambda_{x} u_{x}+A^{\prime \prime} t \lambda_{x}^{2} u_{t}+2 A^{\prime} t \lambda_{x} u_{x t}+\lambda_{x x} A^{\prime} u+\lambda_{x x} A^{\prime} t u_{t}\right) \frac{\partial}{\partial \gamma}
\end{aligned}
$$

so that $\left[V_{5}, V_{6}\right]=0$ only after the augmented system of Equations (23)-(27) is imposed. This observation reminds us of internal symmetries, a notion we now recall following [3]. An internal symmetry of a system of equations $\Xi_{a}=0$ of order $l$ is a vector field $\boldsymbol{W}$ on $S^{(i)}$ such that $\boldsymbol{W}\left(C\left(S^{(i)}\right)\right) \subseteq C\left(S^{(i)}\right)$, in which $C\left(S^{(l)}\right)$ is the differential ideal of contact forms of $S^{(l)}$ generated by the 1 -forms $\theta_{i_{1}, \ldots, i_{k}}^{\alpha}, k=0, \ldots, l-1$, appearing in (3). Certainly, if $W$ were a vector field on the whole jet space $J^{(l)} E$ such that $W\left(C\left(J^{(l)} E\right)\right) \subseteq C\left(J^{(l)} E\right)$ (what is called an external symmetry in [3]), then the restriction of $W$ to $S^{(l)}$ would be an internal symmetry. The question whether there exist internal symmetries that are not restrictions naturally arises. For instance, a subtle result proved in [3] states that if a system of partial differential equations $\Xi_{a}=0$ satisfies a technical condition (dubbed the "descent property"), then every internal symmetry of $\Xi_{a}=0$ is obtained by restriction to $S^{(l)}$ of a first order generalized symmetry of $\Xi_{a}=0$. Now internal symmetries form a Lie algebra on $S^{(l)}$ but if they are not restrictions of external symmetries, there is no reason why they should form a Lie algebra on the jet space $J^{\left({ }^{l}\right.} E$. As (35) shows, the same phenomenon appears in the realm of nonlocal symmetries, reflecting the fact that they 
are defined on coverings of the equation manifold of a (system of) differential equations, and not on some "universal" jet space as it happens with local symmetries [40,42]. Thus, it appears to us that (see also [45]) nonlocal symmetries are really to be thought of as generalizing the class of internal symmetries.

Corollary 2 implies that the nonlocal $\pi$-symmetries (29)-(33) generate a fivedimensional Lie algebra $\mathcal{G}_{5}$. More precisely, if instead of $V_{3}, V_{4}, V_{5}$ we use

$$
e=-\sqrt{2 \lambda} V_{4}, \quad f=\sqrt{2 \lambda} V_{5}, \quad h=-2 \lambda V_{3},
$$

we find the commutators $[h, e]=2 e_{r}[h, f]=-2 f,[e, f]=h$, that is, $e, f, h$ generate a copy of $s l(2)$. Therefore, $\mathcal{G}_{5}$ is isomorphic to the direct sum of $s l(2)$ and the commutative Lie algebra generated by $V_{1}$ and $V_{2}$. Now, if in addition to $V_{1}, \ldots, V_{5}$ we consider also $V_{6}$, we no longer have a finite-dimensional Lie algebra, since the "structure constants" we obtain from the table appearing in Corollary 2 are not constant numbers. In order to analyze the richer structure that results, let us set $A(\lambda)=1$. Then, if instead of $V_{i}, i=$ $1, \ldots, 5$, we consider $f(\lambda) V_{i}$ for some smooth function $f(\lambda)$, we obtain the commutator

$$
\left[f(\lambda) V_{i}, V_{6}\right]=f(\lambda)\left[V_{i}, V_{6}\right]-V_{6}(f(\lambda)) V_{i}=f(\lambda)\left[V_{i}, V_{6}\right]-\lambda f^{\prime}(\lambda) V_{i},
$$

so that if $f(\lambda) V_{i}$ is included in our Lie algebra, so is $\lambda f^{\prime \prime}(\lambda) V_{i}, \lambda^{2} f^{\prime \prime}(\lambda) V_{i}$, and so forth. Thus, taking $f(\lambda)=e^{\lambda}$, for example, we generate an infinite-dimensional Lie algebra of (nonlocal) symmetries to the Camassa-Holm equation in the fixed covering determined by $\gamma, \delta, \beta$, and $\lambda$. More precisely, we observe that the Lie algebra generated by the nonlocal symmetries $V_{1}, \ldots, V_{6}$ contains a semidirect sum of the loop algebra over $\operatorname{sl}(2, \mathbf{R})$ and the centerless Virasoro algebra.

Proposition 2. Let us define the vector fields

$$
T_{n}^{1}=-2 \lambda^{n+1} V_{3}, \quad T_{n}^{2}=-\sqrt{2 \lambda} \lambda^{n} V_{4}, \quad T_{n}^{3}=\sqrt{2 \lambda} \lambda^{n} V_{5}, \quad \text { and } \quad W_{n}=-\lambda^{n} L,
$$

in which $n \in \mathrm{Z}$ and $L=V_{6}$ with $A(\lambda)=1$. Then the following commutation relations hold:

$$
\left[T_{m}^{1}, T_{n}^{2}\right]=2 T_{m+n}^{2}, \quad\left[T_{m}^{1}, T_{n}^{3}\right]=-2 T_{m+n}^{3}, \quad\left[T_{m}^{2}, T_{n}^{3}\right]=T_{m+n}^{1}
$$




$$
\begin{aligned}
& {\left[W_{m}, W_{n}\right]=(m-n) W_{m+n} ;} \\
& {\left[T_{m}^{1}, W_{n}\right]=m T_{m+n}^{1}, \quad\left[T_{m}^{2}, W_{n}\right]=\left(m-\frac{1}{2}\right) T_{m+n}^{2}, \quad\left[T_{m}^{3}, W_{n}\right]=\left(m+\frac{1}{2}\right) T_{m+n}^{3} .}
\end{aligned}
$$

\subsection{Nonlocal symmetries of the associated $\mathrm{CH}$ equation}

We now consider the ACH equation. Define the transformation

$$
p=\sqrt{2 m}, \quad \mathrm{~d} y=p \mathrm{~d} x-p u \mathrm{~d} t, \quad \text { and } \quad \mathrm{d} T=\mathrm{d} t,
$$

and replace in Equation (23) (This formal change of variables makes sense: if $m_{0}(x, t)=$ $u_{0, x x}(x, t)-u_{0}(x, t)$ does not change sign, neither does $m(x, t)=u_{x x}(x, t)-u(x, t)$ as $u(x, t)$ evolves under the $\mathrm{CH}$ flow; see [17] and references therein.). We find

$$
p_{T}=-p^{2} u_{y}, \quad u=\frac{-p^{2}}{2}-\left(\frac{p_{T}}{p}\right)_{y} p
$$

This is Schiff's ACH equation introduced in [49].

Note that transformation (40) is nonlocal: the new independent variable $y$ is a "potential variable" which can be determined from $x_{r} t, p$, and $u$ only through integration in the $(x, t)$ plane (see [8] for information about these "potential" transformations). In particular, it is not an invertible transformation, as explained in [19,49], and so $\mathrm{CH}$ and $\mathrm{ACH}$ are not equivalent equations. Transformations such as $(40)$ can change essentially the character of the nonlinearity of the initial equation (see [37, 51]) so that properties that are not apparent for it may be successfully studied for the transformed equation. This is exactly what happens in the case of the $\mathrm{CH}$ and $\mathrm{ACH}$ equations: Schiff observed in [49] that the ACH equation (41) admits a loop group interpretation, and that this point of view can be used to derive Bäcklund transformations for ACH. Furthermore, M. Fisher and Schiff showed in 1999 (see [19]) that it is possible to compute conservation laws of (41) by means of the classical Miura-Gardner-Kruskal method [32]. On the other hand, as explained in Section 1, a direct construction of conservation laws for $\mathrm{CH}$ was carried out only in 2002 (see [43]) and a Bäcklund-like transformation for $\mathrm{CH}$ has appeared only recently (see [26] and Section 4 of the present paper).

The ACH equation has been also studied for its own sake: its conformal properties have been considered in [28], and an interesting relationship of (41) with the KdV equation has been observed by Hone [27]. Motivated by these works, our 
foregoing remarks, and the observation that potential transformations in general do change symmetry structure (see [40, pp. 121, 122] for a discussion on this point in the context of Burgers' equation, and [55] for relations among (contact) symmetries of a given equation and (a class of) nonlocal symmetries of the corresponding transformed equation), we now study nonlocal symmetries of the ACH equation. First of all, we transform the equations for $\gamma, \delta$, and $\beta$ appearing in Theorem 1 using (40).

\section{Proposition 3.}

(1) The ACH equation admits a pseudo-potential $y$ determined by the compatible equations

$$
\gamma_{Y}=-\frac{1}{2 \lambda p} \gamma^{2}+\frac{p}{2}+\frac{\lambda}{2 p}, \quad \gamma_{T}=\frac{\gamma^{2}}{2}+\frac{p_{T}}{p} \gamma+\lambda u-\frac{1}{2} \lambda^{2}
$$

(2) The ACH equation admits potentials $\delta$ and $\beta$ determined by the compatible equations

$$
\delta_{Y}=\frac{\gamma}{p}, \quad \delta_{T}=\lambda\left(-\frac{p_{T}}{p}-\gamma\right)
$$

and

$$
\beta_{Y}=\frac{p}{2} \mathrm{e}^{\delta / \lambda}, \quad \beta_{T}=\frac{1}{2}\left(-\gamma^{2}+\lambda^{2}\right) \mathrm{e}^{\delta / \lambda} .
$$

We also remark that the transformation (40) implies that

$$
\lambda_{Y}=\lambda_{T}=0
$$

Now, in analogy with the $\mathrm{CH}$ case, we consider symmetry vector fields of the form

$$
W=G^{1} \frac{\partial}{\partial p}+G^{2} \frac{\partial}{\partial u}+H_{1} \frac{\partial}{\partial \gamma}+H_{2} \frac{\partial}{\partial \delta}+H_{3} \frac{\partial}{\partial \beta}+K \frac{\partial}{\partial \lambda},
$$

with $G^{\alpha}, H_{b}, K$ being functions of the variables $y, T, p, u_{,} \gamma, \delta, \beta, \lambda$ and $p_{y}, u_{y}, u_{T}$. We obtain the following theorem. 
Theorem 3. The first-order generalized symmetries of the augmented ACH system (41)-(45), represented by vector fields (46), are

$$
\begin{aligned}
\boldsymbol{W}_{1}= & -p^{2} u_{Y} \frac{\partial}{\partial p}+u_{T} \frac{\partial}{\partial u} \\
& -\left(\frac{\lambda^{2}}{2}-\lambda u-\frac{\gamma^{2}}{2}+p \gamma u_{Y}\right) u_{x} \frac{\partial}{\partial \gamma}-\lambda\left(\gamma-p u_{y}\right) \frac{\partial}{\partial \delta}+\frac{1}{2} \mathrm{e}^{\delta / \lambda}\left(\lambda^{2}-\gamma^{2}\right) \frac{\partial}{\partial \beta} . \\
\boldsymbol{W}_{2}= & p_{Y} \frac{\partial}{\partial p}+u_{Y} \frac{\partial}{\partial u}+\left(\frac{\lambda}{2 p}+\frac{p}{2}-\frac{\gamma^{2}}{2 \lambda p}\right) \frac{\partial}{\partial \gamma}+\frac{\gamma}{p} \frac{\partial}{\partial \delta}+\frac{1}{2} \mathrm{e}^{\delta / \lambda} p \frac{\partial}{\partial \beta}, \\
\boldsymbol{W}_{3}= & \lambda \frac{\partial}{\partial \delta}+\beta \frac{\partial}{\partial \beta}, \\
\boldsymbol{W}_{4}= & \frac{\partial}{\partial \beta}, \\
\boldsymbol{W}_{5}= & 2 \mathrm{e}^{\delta / \lambda} p \gamma \frac{\partial}{\partial p} \\
& +2 \mathrm{e}^{\delta / \lambda} \lambda\left(\gamma-p u_{Y}\right) \frac{\partial}{\partial u}-\mathrm{e}^{\delta / \lambda}\left(\lambda^{2}-\gamma^{2}\right) \frac{\partial}{\partial \gamma}-2 \lambda\left(\mathrm{e}^{\delta / \lambda} \gamma-\beta\right) \frac{\partial}{\partial \delta}+\beta^{2} \frac{\partial}{\partial \beta}, \\
\boldsymbol{W}_{6}= & A(\lambda)\left(p \frac{\partial}{\partial p}+2 u \frac{\partial}{\partial u}+2 \gamma \frac{\partial}{\partial \gamma}+2(\delta-\lambda) \frac{\partial}{\partial \delta}+2 \lambda \frac{\partial}{\partial \lambda}-2 T \frac{\partial}{\partial T}+Y \frac{\partial}{\partial y}\right),
\end{aligned}
$$

where $A(\lambda)$ is an arbitrary function. Consequently, these vector fields are nonlocal symmetries of the associated $\mathrm{CH}$ equation.

The vector field $W_{6}$ is of the form (46) because we can change $\frac{\partial}{\partial T}$ and $\frac{\partial}{\partial y}$ for the equivalent vector fields $-\boldsymbol{W}_{1}$ and $-\boldsymbol{W}_{2}$, respectively.

Corollary 3. The commutator table of the six nonlocal symmetries (47)-(52) of the associated $\mathrm{CH}$ equation is that of Figure 2 whenever $u, p, \gamma, \delta, \beta, \lambda$ solve the augmented $\mathrm{ACH}$ system (41)-(45).

As in the $\mathrm{CH}$ case (see Remark 3) the commutation relations in Figure 2 are valid only on shell. For instance the commutator of $W_{5}, W_{6}$ is, explicitly,

$$
\left[W_{5}, W_{6}\right]=-2 \lambda e^{s / \lambda} A^{\prime}(\lambda) p \lambda_{y}\left(-2 u+Y u_{y}-2 T u_{T}\right) \frac{\partial}{\partial u} .
$$

The symmetries $W_{1}, \ldots, \boldsymbol{W}_{5}$ generate a five-dimensional Lie algebra isomorphic to the direct sum of $s l(2, \mathbf{R})$ and the two-dimensional commutative Lie algebra generated 


\begin{tabular}{c||c|c|c|r|r|c|} 
& $W_{1}$ & $W_{2}$ & $W_{3}$ & $W_{4}$ & $W_{5}$ & $W_{6}{ }^{*}$ \\
\hline \hline$W_{1}$ & & & & & & $-2 B(\lambda) W_{1}$ \\
\hline$W_{2}$ & & & & & & $B(\lambda) W_{2}$ \\
\hline$W_{3}$ & & & & $-W_{4}$ & $W_{5}$ & \\
\hline$W_{4}$ & & & $W_{4}$ & & $2 W_{3}$ & \\
\hline$W_{5}$ & & & $-W_{5}$ & $-2 W_{3}$ & & \\
\hline$W_{6}$ & $2 A(\lambda) W_{1}$ & $-A(\lambda) W_{2}$ & & & & $-2\left[A^{\prime}(\lambda) B(\lambda)-A(\lambda) B^{\prime}(\lambda)\right] \frac{\lambda}{A(\lambda)} W_{6}$ \\
\hline
\end{tabular}

Fig. 2. The commutation table of the ACH nonlocal symmetry algebra. In $W_{6}{ }^{*}$ we have used $B(\lambda)$ instead of $A(\lambda)$.

by $\boldsymbol{W}_{1}, W_{2}$, and addition of $W_{6}$ allows us to construct infinite-dimensional Lie algebras of nonlocal symmetries to the ACH equation. We observe that instead of Proposition 2, in this case we obtain the standard semidirect sum of the loop algebra over $\operatorname{sl}(2, \mathbf{R})$ and the centerless virasoro algebra:

Proposition 4. Let us define the vector fields

$$
T_{n}^{1}=-2 \lambda^{n} \boldsymbol{W}_{3}, \quad T_{n}^{2}=-\lambda^{n} \boldsymbol{W}_{4}, \quad T_{n}^{3}=\lambda^{n} \boldsymbol{W}_{5}, \quad \text { and } \quad V_{n}=-\frac{1}{2} \lambda^{n} L
$$

in which $n \in \mathrm{Z}$ and $L=W_{6}$ with $A(\lambda)=1$. Then the following commutation relations hold:

$$
\begin{aligned}
& {\left[T_{m}^{1}, T_{n}^{2}\right]=2 T_{m+n}^{2}, \quad\left[T_{m}^{1}, T_{n}^{3}\right]=-2 T_{m+n}^{3}, \quad\left[T_{m}^{2}, T_{n}^{3}\right]=T_{m+n}^{1}} \\
& {\left[V_{m}, V_{n}\right]=(m-n) V_{m+n} ;} \\
& {\left[T_{m}^{1}, V_{n}\right]=m T_{m+n}^{1}, \quad\left[T_{m}^{2}, V_{n}\right]=m T_{m+n}^{2} \quad\left[T_{m}^{3}, V_{n}\right]=m T_{m+n}^{2}}
\end{aligned}
$$

\section{Applications}

\subsection{Solving the Camassa-Holm equation via nonlocal symmetries}

In this subsection, we study the flow of the vector field (33), and we use our analysis to obtain explicit solutions to the $\mathrm{CH}$ equation. We focus on (33) because the families of solutions to $\mathrm{CH}$ generated by the vector fields (29), (30), and (34) are simpler to obtain 
(they correspond to shifts of the independent variables and to scaling, respectively) and because the flows of (31) and (32) change the nonlocal variables $\delta$ and $\beta$, but they do not modify the original dependent variables $u$ and $m$.

In agreement with the standard theory of generalized symmetries $[31,40]$, the system of equations we need to consider is

$$
\begin{aligned}
& \frac{\partial u}{\partial \tau}=\gamma \mathrm{e}^{\delta / \lambda}, \\
& \frac{\partial m}{\partial \tau}=\left[\frac{\partial m}{\partial x}+\frac{2}{\lambda} \gamma m\right] \mathrm{e}^{\delta / \lambda}, \\
& \frac{\partial \gamma}{\partial \tau}=m \mathrm{e}^{\delta / \lambda}, \\
& \frac{\partial \delta}{\partial \tau}=\beta, \\
& \frac{\partial \beta}{\partial \tau}=m \mathrm{e}^{2 \delta / \lambda}+\frac{1}{2 \lambda} \beta^{2},
\end{aligned}
$$

with initial conditions

$$
u(X, t, 0)=u_{0}, \quad m(x, t, 0)=m_{0}, \quad \gamma(x, t, 0)=\gamma_{0}, \quad \delta(x, t, 0)=\delta_{0}, \quad \text { and } \quad \beta(x, t, 0)=\beta_{0},
$$

in which $u_{0}(x, t), m_{0}(x, t), \gamma_{0}(x, t), \delta_{0}(x, t)$, and $\beta_{0}(x, t)$ are particular solutions to (23)(26). General theorems on existence, uniqueness, and regularity of solutions to symmetric hyperbolic quasi-linear systems such as $(56)-(60)$ (see $[52$, Chapter 16]) allow us to conclude the following proposition.

Proposition 5. Take $M=S^{1}$ or $M=\mathbf{R}$, and assume that the initial data

$$
u_{0}(x, t), \quad m_{0}(x, t), \quad y_{0}(x, t), \quad \delta_{0}(x, t), \quad \text { and } \quad \beta_{0}(x, t)
$$

belong to the Sobolev space $H^{k}(M)$, with $k>3 / 2$. Then the system (56)-(60) with initial conditions (61) possesses solutions $u(x, t, \tau), m(x, t, \tau), \gamma(x, t, \tau), \delta(x, t, \tau)$, and $\beta(x, t, \tau)$ on an interval $I$ about $\tau=0$, belonging to

$$
L^{\nsim}\left(I, H^{k}(M)\right) \cap \operatorname{Lip}\left(I, H^{k-1}(M)\right)
$$

Furthermore, if the initial data are smooth, then so are the local (in $\tau$ ) solutions $u(x, t, \tau)$, $m(x, t, \tau), y(x, t, \tau), \delta(x, t, \tau)$, and $\beta(x, t, \tau)$. 
This proposition, combined with the results reviewed in Section 2, tells us that if we start with smooth initial conditions (62) globally defined for $x \in S^{1}$ or $x \in \mathbf{R}$, we are able to find (at least for small values of $\tau$ ) families of solutions to the $\mathrm{CH}$ equation also globally defined for $x \in S^{1}$ or $x \in \mathbf{R}$.

Remark 4. We note that Proposition 5 could be applied, for instance, to the interesting special solution

$$
u_{0}(x, t)=c \exp (-|x-c t|)
$$

the standard peakon solution to the $\mathrm{CH}$ equation [10]. However, since a peakon is only a weak solution to $\mathrm{CH}$ (see $[10,14]$ ), standard symmetry theory does not allow us to conclude that the solution $u(x, t, \tau)$ we find from Proposition 5 will also solve $\mathrm{CH}$. We shall not pursue this issue here. The reader is referred to [39], for instance, for information on symmetry theory and generalized solutions to partial differential equations.

We provide explicit formulae for the functions $u(x, t, \tau), m(x, t, \tau), \gamma(x, t, \tau)$, $\delta(x, t, \tau)$, and $\beta(x, t, \tau)$ whose existence is guaranteed by Proposition 5 . The following observation [46] is crucial for simplifying our computations:

Proposition 6. Assume that $m(x, t, \tau)$ and $u(x, t, \tau)$ are related by (23), that is,

$$
m=u_{x X}-u, \quad m_{t}=-m_{X} u-2 m u_{x}
$$

that the functions $\gamma, \delta$, and $\beta$ are defined by means of Equations (24)-(26), and that $m$. $\gamma, \delta$, and $\beta$ satisfy Equations (57)-(60). Then $u(x, t, \tau)$ satisfies Equation (56), namely,

$$
\frac{\partial u}{\partial \tau}=\gamma \mathrm{e}^{\delta / \lambda}
$$

Thus, we do not need to consider the complete vector field (33) in order to study its action on solutions to the system (23)-(26): we can restrict ourselves to the projection of (33) on to the space spanned by $m, \gamma, \delta$, and $\beta$, that is,

$$
V_{\mathrm{pr}}=\mathrm{e}^{\delta / \lambda}\left(\frac{2 m \gamma}{\lambda}+m_{X}\right) \frac{\partial}{\partial m}+\mathrm{e}^{\delta / \lambda} m \frac{\partial}{\partial \gamma}+\beta \frac{\partial}{\partial \delta}+\left(\mathrm{e}^{2 \delta / \lambda} m+\frac{\beta^{2}}{2 \lambda}\right) \frac{\partial}{\partial \beta},
$$

and then find $u$ as a function of the flow parameter $\tau$ simply by using (64). In other words, we change our viewpoint, from thinking of the $\mathrm{CH}$ equation (63) as a differential 
equation for $u(x, t)$, to considering (63) as an integro-differential equation for $m$. This approach has proved to be crucial for the analytic study of the $\mathrm{CH}$ equation: it has been used to determine whether solutions to $\mathrm{CH}$ are global in time or represent breaking waves $[13,15,36]$, and it appears prominently in Lenells' construction of conservation laws [33], in the study of (multi)peakon dynamics and weak solutions [5, 10, 14], in the scattering/inverse scattering approach to $\mathrm{CH}[4,5,17]$, and also in a rigorous proof that the "least action principle" holds for $\mathrm{CH}$ [16].

We concentrate, therefore, on Equations (57)-(60) supplemented with initial conditions

$$
m(x, t, 0)=m_{0}, \quad \gamma(x, t, 0)=\gamma_{0}, \quad \delta(x, t, 0)=\delta_{0}, \quad \text { and } \quad \beta(x, t, 0)=\beta_{0},
$$

in which $m_{0}(x, t), \gamma_{0}(x, t), \delta_{0}(x, t)$, and $\beta_{0}(x, t)$ are particular solutions to (23)-(26). In order to solve this system, we apply a change of independent variables

$$
\xi=f(\tau, x), \quad \eta=g(\tau, x)
$$

so that the system we are interested in becomes

$$
\begin{aligned}
m_{\xi} \xi_{\tau}+m_{\eta} \eta_{\tau} & =\left(m_{\xi} \xi_{X}+m_{\eta} \eta_{X}+\frac{2}{\lambda} \gamma m\right) \mathrm{e}^{\delta / \lambda} \\
\gamma_{\xi} \xi_{\tau}+\gamma_{\eta} \eta_{\tau} & =m \mathrm{e}^{\delta / \lambda} \\
\delta_{\xi} \xi_{\tau}+\delta_{\eta} \eta_{\tau} & =\beta \\
\beta_{\xi} \xi_{\tau}+\beta_{\eta} \eta_{\tau} & =m \mathrm{e}^{2 \delta / \lambda}+\frac{1}{2 \lambda} \beta^{2} .
\end{aligned}
$$

We take $\xi=\tau$ and we choose $\eta$ so that

$$
\eta_{\tau}-\eta_{X} \mathrm{e}^{\delta / \lambda}=0 ; \quad \eta(\tau=0, x)=x
$$

Now we replace back into (67)-(70), and note that the left-hand side of (68) (respectively (69), (70)) contains the derivative of $y$ (respectively $\delta, \beta$ ) with respect to $x$ times the factor $\mathrm{e}^{\delta / \lambda}$. We can simplify (68)-(70) therefore, using Equations (24)-(26). We obtain 
the first-order system

$$
\begin{aligned}
& \frac{\partial m}{\partial \tau}=\frac{2}{\lambda} \gamma(\tau, \eta) m(\tau, \eta) \mathrm{e}^{\delta(\tau, \eta) / \lambda}, \\
& \frac{\partial \gamma}{\partial \tau}=\mathrm{e}^{\delta(\tau, \eta) / \lambda}\left(\frac{1}{2 \lambda} \gamma(\tau, \eta)^{2}-\frac{1}{2} \lambda\right), \\
& \frac{\partial \delta}{\partial \tau}=\beta(\tau, \eta)-\gamma(\tau, \eta) \mathrm{e}^{\delta(\tau, \eta) / \lambda}, \\
& \frac{\partial \beta}{\partial \tau}=\frac{1}{2 \lambda} \beta(\tau, \eta)^{2},
\end{aligned}
$$

which has to be complemented with Equation (71), or, in other words, with the corresponding characteristic equation

$$
\frac{\partial X}{\partial \tau}=-\mathrm{e}^{\delta(\tau, \eta) / \lambda}
$$

Since $\eta$ appears as a parameter in Equations (72)-(76), we can solve them with little difficulty. The following formulae appear in [43], although the foregoing analysis was not explicit in that reference:

Proposition 7. The initial value problem (72)-(76), with initial conditions $\beta_{0}=\beta(0, \eta)$, $\gamma_{0}=\gamma(0, \eta), \delta_{0}=\delta(0, \eta), m_{0}=m(0, \eta)$, and $x_{0}=x(0, \eta)=\eta$, has the solution

$$
\begin{aligned}
& \beta(\tau, \eta)=\frac{1}{B(\eta)} 2 \lambda \beta_{0}, \\
& \gamma(\tau, \eta)=\frac{1}{B(\eta)}\left[\gamma_{0} B(\eta)+\tau \mathrm{e}^{\delta_{0} / \lambda} A^{+}(\eta) A^{-}(\eta)\right], \\
& \delta(\tau, \eta)=\lambda \ln \left|\frac{4 \lambda^{2} \mathrm{e}^{\delta_{0} / \lambda}}{\left(B(\eta)+\tau \mathrm{e}^{\delta_{0} / \lambda} A^{+}(\eta)\right)\left(B(\eta)+\tau \mathrm{e}^{\delta_{0} / \lambda} A^{-}(\eta)\right)}\right|, \\
& m(\tau, \eta)=\frac{1}{B(\eta)^{4}}\left[B(\eta)+\tau \mathrm{e}^{\delta_{0} / \lambda} A^{-}(\eta)\right]^{2}\left[B(\eta)+\tau \mathrm{e}^{\delta_{0} / \lambda} A^{+}(\eta)\right]^{2} m_{0}, \\
& x(\tau, \eta)=\eta+\ln \left|\frac{B(\eta)+\tau \mathrm{e}^{\delta_{0} / \lambda} A^{-}(\eta)}{B(\eta)+\tau \mathrm{e}^{\delta_{0} / \lambda} A^{+}(\eta)}\right|,
\end{aligned}
$$

in which the functions $B, A^{+}$, and $A^{-}$are given by

$$
B(\eta)=-\tau \beta_{0}+2 \lambda, \quad A^{+}(\eta)=\gamma_{0}+\lambda, \quad A^{-}(\eta)=\gamma_{0}-\lambda
$$


Example 1. We consider the Camassa-Holm equation in the form

$$
2 u_{\eta} u_{\eta \eta}+u u_{\eta \eta \eta}=u_{t}-u_{\eta \eta t}+3 u_{\eta} u
$$

so that the "old" space variable is $\eta$. Let us choose an obvious solution of (83), say $u_{0}(\eta, t)=\mathrm{e}^{\eta}$. Then $m_{0}=0$ and the corresponding (pseudo)potentials $\gamma_{0}, \delta_{0}$, and $\beta_{0}$ can be computed by means of (24)-(26). We set

$$
\gamma_{0}=\lambda, \quad \delta_{0}=\lambda \eta-\lambda^{2} t, \quad \beta_{0}=c,
$$

and we use these values as initial conditions for the system (72)-(76). The new space variable $x$ is given by Equation (81). We find

$$
x(\tau, \eta, t)=\eta+\ln \left|\frac{-\tau c+2 \lambda}{-\tau c+2 \lambda+\tau \mathrm{e}^{\eta-\lambda t} 2 \lambda}\right|,
$$

and

$$
m(\tau, \eta, t)=0
$$

while the (pseudo)potentials $\gamma, \delta$, and $\beta$ become

$$
\begin{aligned}
& \gamma(\tau, \eta, t)=\lambda, \\
& \delta(\tau, \eta, t)=\lambda \ln \left|\frac{4 \lambda^{2} \mathrm{e}^{\eta-\lambda t}}{\left(-\tau C+2 \lambda+2 \lambda \tau \mathrm{e}^{\eta-\lambda t}\right)(-\tau C+2 \lambda)}\right| \\
& \beta(\tau, \eta, t)=\frac{2 \lambda c}{-\tau c+2 \lambda} .
\end{aligned}
$$

Now we invert Equation (85). Taking $\beta_{0}=c=0$ and $\tau \geq 0$, we obtain

$$
\mathrm{e}^{x-\eta}=\frac{1}{1+\tau \mathrm{e}^{\eta-\lambda t}}
$$

and it follows that

$$
\eta=x-\ln \left|1-\tau \mathrm{e}^{x-\lambda t}\right| .
$$

This equation allows us to write the (pseudo)potentials $\gamma, \delta$, and $\beta$ as functions of $x, t$, and $\tau$ :

$$
\gamma(x, t, \tau)=\lambda, \quad \delta(x, t, \tau)=\lambda(x-\lambda t), \quad \beta(x, t, \tau)=0,
$$


and it follows that a solution to the Camassa-Holm equation

$$
m=u_{X X}-u, \quad m_{t}=-m_{X} u-2 m u_{x}
$$

is determined by the initial value problem

$$
\frac{\partial u}{\partial \tau}=\gamma(x, t, \tau) \mathrm{e}^{\delta(x, t, \tau) / \lambda}, \quad u(x, t, 0)=\mathrm{e}^{x},
$$

since at $\tau=0$ the independent variables $x$ and $\eta$ coincide. We find

$$
u(x, t, \tau)=\lambda \tau \mathrm{e}^{x-\lambda t}+\mathrm{e}^{x},
$$

a superposition of a travelling wave and the elementary solution $u(x, t)=\mathrm{e}^{x}$.

Example 2. If we take again $u_{0}(\eta, t)=\mathrm{e}^{\eta}$, we obtain a second solution to (91) by using

$$
\gamma_{0}=\frac{\lambda\left(-\lambda+\mathrm{e}^{\eta}\right)}{\mathrm{e}^{\eta}+\lambda} ; \quad \delta_{0}=2 \lambda \ln \left(\mathrm{e}^{\eta}+\lambda\right)-\lambda \eta+\lambda^{2} t ; \quad \beta_{0}=2 \lambda^{2} \mathrm{e}^{\lambda t}
$$

instead of (84). Proceeding as before, we find that the old and new independent variables $\eta$ and $x$ are related by

$$
\eta=\ln \left(\frac{\mathrm{e}^{x}+\tau \lambda^{2} \mathrm{e}^{\lambda t}}{-1+\tau \mathrm{e}^{\lambda t+x}+2 \tau \lambda \mathrm{e}^{\lambda t}}\right)
$$

and that the solution to (91) determined by the initial value problem (92) is

$$
u(x, t, \tau)=-\frac{\mathrm{e}^{X}-\tau \mathrm{e}^{-X+\lambda t} \lambda^{3}}{\tau \lambda \mathrm{e}^{\lambda t}-1}=\frac{-1+\tau\left(\mathrm{e}^{-x+(\lambda / 2) t}\right)^{2} \lambda^{3}}{\tau \lambda \mathrm{e}^{-x+\lambda t}-\mathrm{e}^{-x}} .
$$

We interpret (95) as a nonlinear superposition of travelling wave solutions and the simple stationary solution $u(x, t)=\mathrm{e}^{-x}$.

\subsection{A Darboux transform for the $\mathrm{CH}$ equation}

In this subsection, we present a Darboux-like transformation for the Camassa-Holm equation as an application of the foregoing theory. We follow a method first presented in [50] and then used in [45]. We begin with Equation (80),

$$
m(\tau, \eta)=\frac{1}{B(\eta)^{4}}\left[B(\eta)+\tau e^{\delta_{0} / \lambda} A^{-}(\eta)\right]^{2}\left[B(\eta)+\tau e^{\delta_{0} / \lambda} A^{+}(\eta)\right]^{2} m_{0}
$$


in which $B(\eta)=-\tau \beta_{0}+2 \lambda, A^{+}(\eta)=\gamma_{0}+\lambda$, and $A^{-}(\eta)=\gamma_{0}-\lambda$. Replacing $A^{ \pm}$into this formula and expanding, we obtain

$$
m(\tau, \eta)=\frac{1}{B^{4}}\left[\left(B+\tau \mathrm{e}^{\delta_{0} / \lambda} \gamma_{0}\right)^{2}-\lambda^{2} \tau^{2} \mathrm{e}^{2 \delta_{0} / \lambda}\right]^{2} m_{0},
$$

which may be written, using (25), as

$$
m(\tau, \eta)=\left[\frac{\left(B+\lambda \tau\left(\mathrm{e}^{\delta_{0} / \lambda}\right)_{\eta}\right)^{2}-\lambda^{2} \tau^{2} \mathrm{e}^{2 \delta_{0} / \lambda}}{B^{2}}\right]^{2} m_{0}
$$

Now we replace (26) into this last equation and obtain

$$
m(\tau, \eta)=\left[\frac{\left(B+\lambda \tau\left(\beta_{0 . \eta} / m_{0}\right)_{\eta}\right)^{2}-\lambda^{2} \tau^{2}\left(\beta_{0, \eta} / m_{0}\right)^{2}}{B^{2}}\right]^{2} m_{0}
$$

or, using $B$ instead of $\beta_{0}$,

$$
\begin{aligned}
m(\tau, \eta) & =\left[\left(1-\frac{\lambda}{B}\left(\frac{B_{\eta}}{m_{0}}\right)_{\eta}\right)^{2}-\left(\frac{\lambda B_{\eta}}{B m_{0}}\right)^{2}\right]^{2} m_{0} \\
& =\left[1-\frac{\lambda}{B}\left(\left(\frac{B_{\eta}}{m_{0}}\right)_{\eta}-\frac{B_{\eta}}{m_{0}}\right)\right]^{2}\left[1-\frac{\lambda}{B}\left(\left(\frac{B_{\eta}}{m_{0}}\right)_{\eta}+\frac{B_{\eta}}{m_{0}}\right)\right]^{2} m_{0} .
\end{aligned}
$$

This is the first component of our Darboux transform. Now consider Equation (81) for the new independent variable $x$ in terms of initial data associated with a "seed" solution $u_{0}, m_{0}$ of the $\mathrm{CH}$ equation (23). Proceeding as before, we easily obtain

$$
\begin{aligned}
x(\tau, \eta) & =\eta+\ln \left[\frac{B+\tau \mathrm{e}^{\delta_{0} / \lambda}\left(\gamma_{0}-\lambda\right)}{B+\tau \mathrm{e}^{\delta_{0} / \lambda}\left(\gamma_{0}+\lambda\right)}\right]=\eta+\ln \left[\frac{B+\lambda \tau\left(\mathrm{e}^{\delta_{0} / \lambda}\right)_{\eta}-\lambda \tau \mathrm{e}^{\delta_{0} / \lambda}}{B+\lambda \tau\left(\mathrm{e}^{\delta_{0} / \lambda}\right)_{\eta}+\lambda \tau \mathrm{e}^{\delta_{0} / \lambda}}\right] \\
& =\eta+\ln \left[\frac{B-\lambda\left(\frac{B_{\eta}}{m_{0}}\right)_{\eta}+\lambda\left(\frac{B_{\eta}}{m_{0}}\right)}{B-\lambda\left(\frac{B_{\eta}}{m_{0}}\right)_{\eta}-\lambda\left(\frac{B_{\eta}}{m_{0}}\right)}\right],
\end{aligned}
$$

that is,

$$
x(\tau, \eta)=\eta+\ln \left[\frac{1-\frac{\lambda}{B}\left(\left(\frac{B_{n}}{m_{0}}\right)_{\eta}-\frac{B_{\eta}}{m_{0}}\right)}{1-\frac{\lambda}{B}\left(\left(\frac{B_{\eta}}{m_{0}}\right)_{\eta}+\frac{B_{\eta}}{m_{0}}\right)}\right] .
$$


These computations allow us to prove (we do not write explicitly the dependence on $\tau$ and we omit the subscript o from all formulael the following proposition.

Proposition 8. The Camassa-Holm equation (23), understood as an equation for $m$, is invariant under the transformation $\eta \mapsto x$ and $m(\eta, t) \mapsto \bar{m}(x, t)$, in which

$$
X=X(\eta, t)=\eta+\ln \left[\frac{1-\frac{\lambda}{B}\left(\left(\frac{B_{\eta}}{m}\right)_{\eta}-\frac{B_{\eta}}{m}\right)}{1-\frac{\lambda}{B}\left(\left(\frac{B_{\eta}}{m}\right)_{\eta}+\frac{B_{\eta}}{m}\right)}\right],
$$

and $\bar{m}(x, t)$ is obtained by inverting (98) and replacing into

$$
\bar{m}=\exp [2(x(\eta, t)-\eta)]\left[1-\frac{\lambda}{B}\left(\left(\frac{B_{\eta}}{m}\right)_{\eta}+\frac{B_{\eta}}{m}\right)\right]^{4} m
$$

In Equations (98) and (99), $B=B(\eta)=-\tau \beta(\eta, t)+2 \lambda$, the functions $m(\eta, t)$ and $\beta(\eta, t)$ are related by

$$
m=\lambda \frac{\partial^{2}}{\partial \eta^{2}} \ln \left(\frac{\beta_{\eta}}{m}\right)+\frac{\lambda}{2}\left[\frac{\partial}{\partial \eta} \ln \left(\frac{\beta_{\eta}}{m}\right)\right]^{2}-\frac{\lambda}{2}
$$

and $\beta(\eta, t)$ is a solution to the equation obtained from replacing (100) into

$$
\beta_{t}=\frac{\beta_{\eta}}{m}\left(-\frac{\lambda^{2}}{2}\left[\frac{\partial}{\partial \eta} \ln \left(\frac{\beta_{\eta}}{m}\right)\right]^{2}+\frac{\lambda^{2}}{2}-u m\right) .
$$

Proof. We just derived Equation (98). Equation (99) is then obtained by replacing (98) into (96). In order to find Equation (100), we write $\delta=\lambda \ln \left(\beta_{\eta} / m\right)$ using the first equation in (26), and then we substitute into the first equation appearing in (24). Finally, Equation (101) is obtained by writing $\delta$ and $\delta_{\eta}$ in terms of $\beta$ as before and substituting into the second equation in (26).

\subsection{A recursion operator for the $\mathrm{CH}$ equation}

In this subsection, we remark that we can recover the recursion operator for the Camassa-Holm equation $[10,20]$ using the shadow $y \mathrm{e}^{(1 / \lambda) \delta} \frac{\partial}{\partial u}$ of the nonlocal 
symmetry (33). Let us define $\psi$ by means of

$$
y=2 \lambda \frac{\psi_{X}}{\psi}=2 \lambda \ln (\psi)_{x}
$$

in which $\gamma$ is a solution to the pseudo-potential equations (24). The function $\psi$ solves the second-order linear problem

$$
\psi_{X X}=\left(\frac{1}{2 \lambda} m+\frac{1}{4}\right) \psi
$$

appearing in the scattering/inverse scattering analysis of the $\mathrm{CH}$ equation [4, 10]. We also obtain an equation for $\psi_{t}$ from the second equation of (24), but we will not need it for what follows. We write the potential $\delta$ in terms of $\psi$ as

$$
\delta=2 \lambda \ln (\psi),
$$

and therefore the function $G=\gamma \mathrm{e}^{(1 / \lambda) \delta}$ becomes

$$
G=2 \lambda \frac{\psi_{x}}{\psi} \exp ((1 / \lambda) 2 \lambda \ln (\psi))=2 \lambda \frac{\psi_{x}}{\psi}[\exp (\ln (\psi))]^{2}=2 \lambda \psi_{X} \psi=\lambda\left(\psi^{2}\right)_{X}
$$

so that our shadow $G$ is in fact a "squared eigenfunction"! Our goal is to obtain an eigenvalue problem for $G$ using (103). First of all we note that

$$
D_{x}^{-1} G=\lambda \psi^{2}, \quad D_{X} G=2 \lambda\left(\psi_{x}^{2}+\psi \psi_{X X}\right), \quad D_{x}^{2} G=2 \lambda\left(3 \psi_{X} \psi_{X X}+\psi \psi_{X X X}\right)
$$

We multiply (103) by $2 \lambda \psi_{x}$ and we obtain

$$
2 \lambda \psi_{X} \psi_{X X}=\left(\frac{1}{2 \lambda} m+\frac{1}{4}\right) G
$$

On the other hand, the third equation appearing in (105) implies that

$$
2 \lambda \psi_{X} \psi_{X x}=\frac{1}{3}\left(D_{x}^{2} G-2 \lambda \psi \psi_{x x x}\right)
$$

and we can compute the term $2 \lambda \psi \psi_{x x x}$ appearing in this equation taking $x$-derivative of (103) and multiplying the resulting equation by $2 \lambda \psi$. We obtain, using again (105),

$$
2 \lambda \psi \psi_{X X X}=\frac{1}{\lambda} m_{X} D_{x}^{-1} G+\left(\frac{1}{2 \lambda} m+\frac{1}{4}\right) G,
$$


and therefore (107) becomes

$$
2 \lambda \psi_{x} \psi_{x x}=\frac{1}{3}\left(D_{X}^{2} G-\frac{1}{\lambda} m_{x} D_{X}^{-1} G-\left(\frac{1}{2 \lambda} m+\frac{1}{4}\right) G\right) .
$$

Replacing into Equation (106), we find the following equation for the shadow $G$ :

$$
D_{x}^{2} G-\frac{1}{\lambda} m_{x} D_{x}^{-1} G-\frac{2}{\lambda} m G=G .
$$

Equivalently, we can write this equation as

$$
\left(D_{x}^{2}-1\right)^{-1}\left(m_{X} D_{x}^{-1}+2 m\right) G=\lambda G
$$

This is the eigenvalue equation for the squared eigenfunction $G$ which we were trying to find. The formal pseudo-differential operator $\left(D_{X}^{2}-1\right)^{-1}\left(m_{X} D_{X}^{-1}+2 m\right)$ is precisely the recursion operator for the Camassa-Holm equation [20].

\subsection{Solving the ACH equation via nonlocal symmetries}

Solutions of the ACH equation

$$
p_{T}=-p^{2} u_{y}, \quad u=\frac{-p^{2}}{2}-\left(\frac{p_{T}}{p}\right)_{y} p
$$

have been obtained by Schiff [49] and Hone [27], independently of the relation between $\mathrm{CH}$ and $\mathrm{ACH}$. In particular, Hone shows in [27] how to obtain solutions to the ACH equation from $\tau$-functions of the KdV hierarchy. It is of interest to generate solutions to $\mathrm{ACH}$ without using the potential transformation (40), because this change of variables can be applied only to positive (or negative) solutions to the Camassa-Holm equation; see [49]. We note that our elementary solutions (93) and (95) change sign (depending on the values of the parameters $\lambda$ and $\tau$ ), and that a formal application of (40) to them only yields trivial solutions.

We generate solutions to $\mathrm{ACH}$ using nonlocal symmetries. We consider solutions generated by the flow of the vector field (51), by reasons entirely analogous to the ones given at the beginning of Section 4.1.

The flow of (51) is determined by the system of equations

$$
\frac{\partial p}{\partial \tau}=2 e^{\delta / \lambda} p \gamma
$$




$$
\begin{aligned}
& \frac{\partial u}{\partial \tau}=2 \lambda \mathrm{e}^{\delta / \lambda}\left(\gamma-p \frac{\partial u}{\partial y}\right), \\
& \frac{\partial \gamma}{\partial \tau}=-\mathrm{e}^{\delta / \lambda}\left(\lambda^{2}-\gamma^{2}\right), \\
& \frac{\partial \delta}{\partial \tau}=-2 \lambda\left(\mathrm{e}^{\delta / \lambda} \gamma-\beta\right), \\
& \frac{\partial \beta}{\partial \tau}=\beta^{2},
\end{aligned}
$$

with initial conditions $u(y, T, 0)=u_{0}, p(y, T, 0)=p_{0}, \gamma(y, T, 0)=\gamma_{0}, \delta(y, T, 0)=\delta_{0}$, and $\beta(y, T, 0)=\beta_{0}$. We easily find

$$
\begin{aligned}
& \beta(\tau)=\frac{\beta_{0}}{1-\tau \beta_{0}}, \\
& \gamma(\tau)=\frac{\tau\left(\lambda^{2} \omega_{0}-\omega \gamma_{0}^{2}+\gamma_{0} \beta_{0}\right)-\gamma_{0}}{\tau \beta_{0}-1}, \\
& \delta(\tau)=\delta_{0}-\lambda \ln \left[\left(1-\tau \beta_{0}+\omega_{0} \gamma_{0} \tau-\tau \lambda \omega_{0}\right)\left(1-\tau \beta_{0}+\omega_{0} \gamma_{0} \tau+\tau \lambda \omega_{0}\right)\right], \\
& p(\tau)=\frac{p_{0}\left(1-\tau \beta_{0}+\omega_{0} \gamma_{0} \tau-\tau \lambda \omega_{0}\right)\left(1-\tau \beta_{0}+\omega_{0} \gamma_{0} \tau+\tau \lambda \omega_{0}\right)}{\left(-1+\tau \beta_{0}\right)^{2}},
\end{aligned}
$$

in which $\omega_{0}=\mathrm{e}^{\delta_{0} / \lambda}$. It remains to obtain $u(\tau)$. In actual fact, it is possible to obtain $u(\tau)$ directly from the ACH equation (109) without solving Equation (111), as this equation is a consequence of the augmented ACH system (41)-(44) and the flow Equations (110) and (1 12)-(114):

Proposition 9. Assume that $p, u$ and the functions $\gamma, \delta$, and $\beta$ satisfy the compatible Equations (41)-(44), (110), and (112)-(114). Then $u$ satisfies Equation (111), namely,

$$
\frac{\partial u}{\partial \tau}=2 \lambda \mathrm{e}^{\delta / \lambda}\left(\gamma-p \frac{\partial u}{\partial y}\right)
$$

Proof. We take the derivative with respect to $\tau$ in Equation (42) for $\gamma_{T}$ and simplify using (43), (44) and the flow Equations (110) and (112)-(114). We obtain

$$
\gamma_{T \tau}=2 \lambda u \gamma \mathrm{e}^{\delta / \lambda}-\lambda^{2} \frac{p_{T}}{p} \mathrm{e}^{\delta / \lambda}+\gamma^{2} \frac{p_{T}}{p} \mathrm{e}^{\delta / \lambda}-2 \lambda^{2} \gamma \mathrm{e}^{\delta / \lambda}+\lambda u_{\mathrm{\tau}}
$$


On the other hand, taking the derivative with respect to $T$ in Equation (112) yields

$$
\gamma_{\tau} T=\lambda^{2} \frac{p_{T}}{p} \mathrm{e}^{\delta / \lambda}+\gamma^{2} \frac{p_{T}}{p} \mathrm{e}^{\delta / \lambda}+2 \lambda y u \mathrm{e}^{\delta / \lambda}
$$

Equating (120) and (121), we find

$$
2 \lambda \gamma \mathrm{e}^{s / \lambda}+2 \lambda \mathrm{e}^{s / \lambda} \frac{p_{T}}{p}=\frac{\partial u}{\partial \tau}
$$

and use of (41) yields (119).

Example 3. We take $p_{0}=c$, in which $c$ is a constant different from zero. Equation (41) tells us that $u_{0}=-c^{2} / 2$. We compute $\gamma_{0}, \delta_{0}$, and $\beta_{0}$ by means of (42)-(44) and obtain

$$
\begin{aligned}
\gamma_{0}(y, T) & =\mu \lambda, \\
\delta_{0} & =\mu \lambda\left(\frac{y}{c}-\lambda T\right), \\
\beta_{0} & =\frac{c^{2}}{2 \mu} \exp \left[\mu\left(\frac{Y}{c}-\lambda T\right)\right],
\end{aligned}
$$

in which $\mu=\sqrt{\lambda c^{2}+\lambda^{2}} / \lambda$. Equation (118) then implies that

$$
p(y, T, \tau)=c \frac{\left(1+\tau\left(-\frac{c^{2}}{2 \mu}+\lambda \mu+\lambda\right) \mathrm{e}^{\mu\left(\frac{\gamma}{c}-\lambda T\right)}\right)\left(1+\tau\left(-\frac{c^{2}}{2 \mu}+\lambda \mu-\lambda\right) \mathrm{e}^{\mu\left(\frac{\underline{y}}{c}-\lambda T\right)}\right)}{\left(-1+\tau \frac{c^{2}}{2 \mu} \mathrm{e}^{\mu(\underline{\underline{Y}}-\lambda T)}\right)^{2}}
$$

solves the ACH equation (109).

\subsection{Darboux transforms for the ACH equation}

In this subsection we obtain two Darboux transforms for the ACH equation (109). The second transformation is obtained via a reasoning analogous to the one used for the $\mathrm{CH}$ equation, while the first Darboux transform does not have an analog in the $\mathrm{CH}$ theory (What can be proved in the $\mathrm{CH}$ case following our first method is studied in the paper [22]). We finish our work pointing out that combination of our two transformations allows us to exhibit a nonlinear superposition rule for ACH. 
Let us consider the quadratic pseudo-potential $\gamma(Y, T)$ defined by Equations (42), namely,

$$
\gamma_{Y}=-\frac{1}{2 \lambda p} \gamma^{2}+\frac{p}{2}+\frac{\lambda}{2 p}, \quad \gamma_{T}=\frac{\gamma^{2}}{2}+\frac{p_{T}}{p} \gamma+\lambda u-\frac{1}{2} \lambda^{2} .
$$

Computing $p(y, T)$ from the first equation in (126), we obtain

$$
p(Y, T)=\frac{\partial}{\partial y} \gamma(y, T)+\sqrt{\left(\frac{\partial}{\partial y} \gamma(y, T)\right)^{2}+\frac{\gamma(y, T)^{2}}{\lambda}-\lambda} .
$$

and replacing (127) into the equation for $\gamma_{T}$, we find a (fairly complicated) differential equation for $\gamma$, which, however, can be studied using MAPLE: it can be checked that this equation is invariant under the change

$$
y \mapsto-y, \quad \sqrt{\lambda} \mapsto-\sqrt{\lambda}
$$

(We note that this method yields, in the $\mathrm{CH}$ case, a nonlocal equation for the $\mathrm{CH}$ pseudopotential; see [22]).

Equation (127), however, is not invariant under the change (127a). Instead, we obtain that

$$
\bar{p}(y, T)=-\frac{\partial}{\partial y} \gamma(Y, T)+\sqrt{\left(\frac{\partial}{\partial y} \gamma(Y, T)\right)^{2}+\frac{\gamma(y, T)^{2}}{\lambda}-\lambda}
$$

is also a solution to ACH. Subtracting (127) and (128), we find the following proposition.

Proposition 10. If $\gamma(y, T)$ is determined by Equations $(42)$, and $p(y, T)$ is a solution to the ACH equation (41), then so is

$$
\bar{p}(y, T)=p(y, T)-2 \frac{\partial y}{\partial y}(Y, T) .
$$

This transformation was found by Schiff in [49] using loop groups, and it has been re-derived by Hone from a relationship between $\mathrm{KdV}$ and $\mathrm{ACH}$ found by him in [27]. of some interest is the fact that it depends only on the existence of a quadratic pseudopotential for $\mathrm{ACH}$.

Now we use the flow of the vector field (51) as given by formulas (115)-(118) to obtain a second Darboux transform for ACH: 
Proposition 11. Assume that $p(y, T)$ solves the ACH equation (4l), and that $\beta(y, T)$ is a solution to

$$
\frac{\beta_{T}}{\beta_{y}}=\frac{-\gamma^{2}+\lambda^{2}}{p}
$$

in which $\gamma$ is a solution to the compatible system (42). Then the function $\bar{p}(y, T)$ given by

$$
\bar{p}=p\left(-1+\frac{2 \tau \lambda p}{-1+\tau \beta}\left(\frac{\beta_{Y}}{p}\right)_{Y}\right)^{2}-\frac{4 \lambda^{2} \tau^{2}}{(-1+\tau \beta)^{2}} \frac{\beta_{Y}^{2}}{p}
$$

also solves (41).

Proof. We start with Equation (127) for $p(y, T)$ and we write it in the form

$$
p=p_{0}\left(-1+\frac{\omega_{0} \gamma_{0} \tau}{-1+\tau \beta_{0}}\right)^{2}-\frac{\tau^{2} \lambda^{2}}{\left(-1+\tau \beta_{0}\right)^{2}} p_{0} \omega_{0}^{2}
$$

Now we use Equations (43) and $\omega_{0}=e^{\delta_{0} / \lambda}$ to write (131) as

$$
p=p_{0}\left(-1+\frac{\tau \lambda p_{0}\left(\mathrm{e}^{\delta_{0} / \lambda}\right)_{Y}}{-1+\tau \beta_{0}}\right)^{2}-\frac{\tau^{2} \lambda^{2}}{\left(-1+\tau \beta_{0}\right)^{2}} p_{0} \mathrm{e}^{2 \delta_{0} / \lambda}
$$

and we find (130) from this equation by writing $\mathrm{e}^{\delta_{0} / \lambda}$ in terms of $\beta_{0, y}$ and $p_{0}$ using the first equation in (44), changing $p$ to $\bar{p}$, and then dropping the subindex 0 . Finally, Equation (129) is obtained directly from the second equation in (44), simply using the identity $\mathrm{e}^{\delta_{0} / \lambda}=2 \beta_{y} / p$ as above.

Finally, we note that the first equation in (126) implies that Equation (129) can be written as

$$
\frac{\beta_{T}}{\beta_{Y}}=-\lambda\left(p-2 \gamma_{Y}\right)
$$

and Proposition 10 says that $\hat{p}=p-2 \gamma_{y}$ is a new solution to the ACH equation. We therefore interpret Proposition 11 as providing us with a nonlinear superposition rule for $\mathrm{ACH}$.

\section{Funding}

R.H.H.'s research was partially financed by the Universidad Politécnica de Madrid, Programa de Colaboración con Latinoamérica ref. AL07-PID-056 and AL08-PID-49. E.G.R.'s research is 
partially supported by the Fondo Nacional de Desarrollo Científico y Tecnológico (FONDECYT) grant \#1070191, and by the Research Ring "Anillo Ecuaciones Asociadas a Reticulados" financed by the World Bank through the Programa Bicentenario de Ciencia y Tecnología.

\section{References}

[1] Adler, V. E. "Lie-algebraic approach to nonlocal symmetries of integrable systems." Teoreticheskaya i Matematicheskaya Fizika 89, no. 3 (1991): 323-36.

[2] Akhatov, I. Sh., R. K. Gazizov, and N. Kh. Ibragimov. "Nonlocal symmetries. Heuristic approach." Journal of Soviet Mathematics 55, no. 1 (1991): 1401-50.

[3] Anderson, I. M., N. Kamran, and P. J. Olver. "Internal, external and generalized symmetries." Advances in Mathematics 100, no. 1 (1993): 53-100.

[4] Beals, R., D. H. Sattinger, and J. Szmigielski. "Acoustic scattering and the extended Korteweg-de Vries hierarchy." Advances in Mathematics 140, no. 2 (1998): 190-206.

[5] Beals, R., D. H. Sattinger, and J. Szmigielski. "Multipeakons and the classical moment problem." Advances in Mathematics 154, no. 2 (2000): 229-57.

[6] van Bemmelen, T. "Applications of coverings and non-local symmetries." Journal of Physics A: Mathematical and General 26, no. 22 (1993): 6409-20.

[7] Bernshtein, I. N. and B. I. Rozenfel'd. "Homogeneous spaces of infinite-dimensional Lie algebras and characteristic classes of foliations." Russian Mathematical Surveys 28, no. 4 (1973): $107-42$.

[8] Bluman, G. W., A. F. Cheviakov, and S. G. Anco. Applications of Symmetry Methods to Partial Differential Equations. Springer Series in Applied Mathematical Sciences 168. New York: Springer 2010.

[9] Bluman, G. W. and S. Kumei. "Symmetry-based algorithms to relate partial differential equations. II. Linearization by nonlocal symmetries." European Journal of Applied Mathematics 1, no. 1 (1990): 217-23.

[10] Camassa, R. and D. D. Holm. "An integrable shallow water equation with peaked solitons." Physical Review Letters 71, no. 11 (1993): 1661-4.

[11] Camassa, R., D. D. Holm, and J. M. Hyman. "A new integrable shallow water equation." Advances in Applied Mathematics 31 (1994): 1-33.

[12] Chern, S. S. and K. Tenenblat. "Pseudo-spherical surfaces and evolution equations." Studies in Applied Mathematics 74, no. 1 (1986): 55-83.

[13] Constantin, A. "Existence of permanent and breaking waves for a shallow water equation: a geometric approach." Annales de l'Institut Fourier (Grenoble) 50, no. 2 (2000): 321-62.

[14] Constantin, A. and J. Escher. "Global weak solutions for a shallow water equation." Indiana University Mathematics Journal 47, no. 4 (1998): 1527-45.

[15] Constantin, A. and J. Escher. "Wave breaking for nonlinear nonlocal shallow water equations." Acta Mathematica 181, no. 2 (1998): 229-43.

[16] Constantin, A. and B. Kolev. "Geodesic flow on the diffeomorphism group of the circle." Commentarii Mathematici Helvetici 78, no. 4 (2003): 787-804. 
[17] Constantin, A. and H. P. McKean. "A shallow water equation on the circle." Communications on Pure and Applied Mathematics LII, no. 8 (1999): 949-82.

[18] Dodd, R. K. "The general prolongation formulae for nonlocal symmetries." Physics Letters A 195, no. 2 (1994): 125-7.

[19] Fisher, M. and J. Schiff. "The Camassa Holm equation: conserved quantities and the initial value problem." Physics Letters A 259, no. 5 (1999): 371-6.

[20] Fuchssteiner, B. "Some tricks from the symmetry-toolbox for nonlinear equations." Physica D 95, no. 3-4 (1996): 229-43.

[21] Galas, F. "New nonlocal symmetries with pseudopotentials." Journal of Physics A 25, no. 15 (1992): L981-6.

[22] Górka, P. and E. G. Reyes. "The modified Camassa-Holm equation." International Mathematics Research Notices, September 2010, doi:10.1093/imrn/rnq 163.

[23] Guthrie, G. A. "More non-local symmetries of the KdV equation." Joumal of Physics A: Mathematical and General 26, no. 18 (1993): L905-8.

[24] Guthrie, G. A. and M. S. Hickman. "Nonlocal symmetries of the KdV equation." Journal of Mathematical Physics 34, no. 1 (1993): 193-205.

[25] Hernández Heredero, R. "Mathematica Packages for the Computation of Symmetries." http://www.euitt.upm.es/departamentos/mat/rafahh/Research.html.

[26] Hernández Heredero, R. and E. G. Reyes. "Nonlocal symmetries and a Darboux transformation for the Camassa-Holm equation." Journal of Physics A: Mathematics and Theoretical 42, no. 18 (2009): 182002 (Fast Track Communication, 9 pp).

[27] Hone, A. N. W. "The associated Camassa-Holm equation and the KdV equation." Journal of Physics A: Mathematical and General 32, no. 27 (1999): L307-14.

[28] Ivanov, R. "Conformal properties and Bäcklund transform for the associated Camassa-Holm equation." Physics Letters A 345, no. 1-3 (2005): 112-8.

[29] Kirnasov, E. G. "Wahlquist-Estabrook type coverings over the heat equation." Matematicheskie Zametki 42, no. 3 (1987): 422-34.

[30] Krasil'shchik, I. S. and A. M. Vinogradov. "Nonlocal trends in the geometry of differential equations: Symmetries, conservation laws and Bäcklund transformations." Acta Applicandae Mathematicae 15, no. 1-2 (1989): 161-209.

[31] Krasil'shchik, I. S. and A. M. Vinogradov (Eds). Symmetries and Conservation Laws for Differential Equations of Mathematical Physics. Translations of Mathematical Monographs 182. Providence, RI: AMS, 1999.

[32] Kruskal, M. D., R. M. Miura, and C. S. Gardner. "Korteweg-de Vries equation and generalizations. V. Uniqueness and nonexistence of polynomial conservation laws." Journal of Mathematical Physics 11, no. 3 (1970): 952-60.

[33] Lenells, J. "Conservation laws of the Camassa-Holm equation." Journal of Physics A: Mathematical and General 38, no. 4 (2005): 869-80.

[34] Leo, M., R. A. Leo, G. Soliani, and P. Tempesta. "Non-local Bäcklund Transformations for Harry Dym and Korteweg-de Vries Equations." In Nonlinearity, Integrability and all that: 
Twenty Years After NEEDS'79, edited by M. Boiti, L. Martina, F. Pempinelli, B. Prinari, and G. Soliani, 318-24. Singapore: World Scientific Publishing, 2000.

[35] Leo, M., R. Leo, G. Soliani, and P. Tempesta. "On the relation between Lie symmetries and prolongation structures of nonlinear field equations-non-local symmetries." Progress of Theoretical Physics 105, no. 1 (2001): 77-97.

[36] Li, Y. A. and P. J. Olver, "Well-posedness and blow-up solutions for an integrable nonlinearly dispersive model wave equation." Journal of Differential Equations 162, no. 1 (2000): 27-63.

[37] Mikhailov, A. V., A. B. Shabat, and R. I. Yamilov. "The symmetry approach to the classification of non-linear equations. Complete lists of integrable systems." Russian Mathematical Surveys 42, no. 4 (1987): 1-63.

[38] Molino, P. Systèmes différentiels extérieurs et équations d'évolution. Elective Course in Differential Geometry, 1980-1981, 70-218. Montpellier: Univ. Sci. Tech. Languedoc, 1981.

[39] Oberguggenberger, M. "Symmetry groups, nonlinear partial differential equations, and generalized functions." Contemporary Mathematics 285 (2001): 101-10.

[40] Olver, P. J. Applications of Lie Groups to Differential Equations, 2nd ed. New York: Springer, 1993.

[41] Olver, P. J. "Nonlocal symmetries and ghosts." In New Trends in Integrability and Partial Solvability, edited by A.B. Shabat, 199-215. Dordrecht, Netherlands: Kluwer Acad. Publ., 2004.

[42] Olver, P. J., J. Sanders, and J.-P. Wang. "Ghost symmetries." Journal of Nonlinear Mathematical Physics 9, Supplement 1 (2002): 164-72.

[43] Reyes, E. G. "Geometric integrability of the Camassa-Holm equation." Letters in Mathematical Physics 59, no. 2 (2002): 117-31.

[44] Reyes, E. G. "The soliton content of the Camassa-Holm and Hunter-Saxton equations." In Proceedings of the Fourth International Conference on Symmetry in Nonlinear Mathematical Physics, edited by A. G. Nikitin, V. M. Boyko, and R. O. Popovych, 201-8. Kyiv: Proceedings of the Institute of Mathematics of the NAS of Ukraine 43, 2002.

[45] Reyes, E. G. "Nonlocal symmetries and the Kaup-Kupershmidt equation." Journal of Mathematical Physics 46, no. 7 (2005): 073507, 19 pp.

[46] Reyes, E. G. "On nonlocal symmetries of some shallow water equations." Journal of Physics A: Mathematical and Theoretical 40, no. 17 (2007): 4467-76.

[47] Sasaki, R. "Pseudopotentials for the general AKNS system." Physics Letters A 73, no. 2 (1979): 77-80.

[48] Schiff, J. "Zero curvature formulations of dual hierarchies." Journal of Mathematical Physics 37, no. 4 (1996): 1928-38.

[49] Schiff, J. "The Camassa-Holm equation: a loop group approach." Physica $D$ 121, no. 1-2 (1998): 24-43.

[50] Schiff, J. "Symmetries of KdV and Loop Groups." Preprint, solv-int/9606004 Los Alamos ePrint archive.

[51] Sokolov, V. V. "Pseudosymmetries and Differential Substitutions." Functional Analysis and its Applications 22, no. 2 (1988): 121-9. 
[52] Taylor, M. E. Partial Differential Equations II. Nonlinear Equations. New York: Springer, 1996.

[53] Vinogradov, A. M. and I. S. Krasil'shchik. "A method of calculating higher symmetries of nonlinear evolutionary equations, and nonlocal symmetries." Doklady Akademii Nauk SSSR 253, no. 6 (1980): 1289-93.

[54] Wahlquist, H. D. and F. B. Eastbrook. "Prolongation structures of nonlinear equations." Journal of Mathematical Physics 16, no. 1 (1975): 1-7.

[55] Zhdanov. R. "On relation between potential and contact symmetries of evolution equations." Journal of Mathematical Physics 50 (2009): 053522, doi:10.1063/1.3138147. 